

\section{The homotopy category of injectives}

\section{Amnon Neeman}

Krause studied the homotopy category $\mathbf{K}(\operatorname{Inj} \mathscr{A})$ of complexes of injectives in a locally noetherian Grothendieck abelian category $\mathscr{A}$. Because $\mathscr{A}$ is assumed locally noetherian, we know that arbitrary direct sums of injectives are injective, and hence, the category $\mathbf{K}(\operatorname{Inj} \mathscr{A})$ has coproducts. It turns out that $\mathbf{K}(\operatorname{Inj} \mathscr{A})$ is compactly generated, and Krause studies the relation between the compact objects in $\mathbf{K}(\operatorname{Inj} \mathscr{A})$, the derived category $\mathbf{D}(\mathscr{A})$, and the category $\mathbf{K}_{\mathrm{ac}}(\operatorname{Inj} \mathscr{A})$ of acyclic objects in $\mathbf{K}(\operatorname{Inj} \mathscr{A})$.

We wish to understand what happens in the nonnoetherian case, and this paper begins the study. We prove that, for an arbitrary Grothendieck abelian category $\mathcal{A}$, the category $\mathbf{K}(\operatorname{Inj} \mathscr{A})$ has coproducts and is $\mu$-compactly generated for some sufficiently large $\mu$.

The existence of coproducts follows easily from a result of Krause: the point is that the natural inclusion of $\mathbf{K}(\operatorname{Inj} \mathscr{A})$ into $\mathbf{K}(\mathscr{A})$ has a left adjoint and the existence of coproducts is a formal corollary. But in order to prove anything about these coproducts, for example the $\mu$-compact generation, we need to have a handle on this adjoint.

Also interesting is the counterexample at the end of the article: we produce a locally noetherian Grothendieck abelian category in which products of acyclic complexes need not be acyclic. It follows that $\mathbf{D}(\mathscr{A})$ is not compactly generated. I believe this is the first known example of such a thing.

Introduction

1. Cardinality estimates in Grothendieck abelian categories

2. An adjoint to the inclusion $\mathrm{K}(\operatorname{Inj} \mathscr{A}) \hookrightarrow \mathrm{K}(\mathscr{A})$

3. The $\mu$-compact generation of $\mathrm{K}(\operatorname{Inj} \mathscr{A})$

4. The failure of recollement

References

This research was partly supported by the Australian Research Council. The author would like to thank an anonymous referee for several suggestions of improvements to the manuscript.

MSC2010: primary 13D09; secondary 08B30.

Keywords: locally presentable categories, well generated categories, homotopy categories of injectives. 


\section{Introduction}

The starting point of this investigation is the article by Krause [2005], in which he studied the homotopy category $\mathbf{K}(\operatorname{Inj} \mathscr{A})$ of complexes of injectives in a locally noetherian Grothendieck abelian category $\mathscr{A}$. It turns out that $\mathbf{K}(\operatorname{Inj} \mathscr{A})$ is compactly generated, the compact objects being injective resolutions of bounded complexes of noetherian objects. In symbols, we have an equivalence $\mathbf{K}(\operatorname{Inj} \mathscr{A})^{c} \cong \mathbf{D}^{b}($ noeth $\mathscr{A})$. We can consider the sequence of functors

$$
\mathbf{K}_{\mathrm{ac}}(\operatorname{Inj} \mathscr{A}) \stackrel{J}{\rightarrow} \mathbf{K}(\operatorname{Inj} \mathscr{A}) \stackrel{Q}{\rightarrow} \mathbf{D}(\mathscr{A}),
$$

which expresses $\mathbf{D}(\mathscr{A})$ as the quotient of $\mathbf{K}(\operatorname{Inj} \mathscr{A})$ by the subcategory of acyclics $\mathbf{K}_{\mathrm{ac}}(\operatorname{Inj} \mathscr{A}) \subset \mathbf{K}(\operatorname{Inj} \mathscr{A})$. It is not hard to prove that this is a localization sequence: the functors $J$ and $Q$ have right adjoints, denoted $J_{\rho}$ and $Q_{\rho}$, respectively. Not so formal is that, as long as $\mathbf{D}(\mathscr{A})$ is compactly generated, the functors $J$ and $Q$ also have left adjoints $J_{\lambda}$ and $Q_{\lambda}$ turning this into a recollement. If we restrict $J_{\lambda}$ and $Q_{\lambda}$ to the subcategories of compact objects, then we have functors

$$
\mathbf{D}(\mathscr{A})^{c} \stackrel{Q_{\lambda}}{\longrightarrow} \mathbf{K}(\operatorname{Inj} \mathscr{A})^{c} \stackrel{J_{\lambda}}{\longrightarrow} \mathbf{K}_{\mathrm{ac}}(\operatorname{Inj} \mathscr{A})^{c},
$$

which allow us to identify $\mathbf{K}_{\mathrm{ac}}(\operatorname{Inj} \mathscr{A})^{c}$ as the idempotent completion of the Verdier quotient $\mathbf{K}(\operatorname{Inj} \mathscr{A})^{c} / \mathbf{D}(\mathscr{A})^{c}$.

In the generality above, where $\mathscr{A}$ is an arbitrary locally noetherian Grothendieck category, we understand the compact objects only in $\mathbf{K}(\operatorname{Inj} \mathscr{A})$, where we have $\mathbf{K}(\operatorname{Inj} \mathscr{A})^{c}=\mathbf{D}^{b}$ (noeth $\left.\mathscr{A}\right)$. But in examples, we sometimes also know $\mathbf{D}(\mathscr{A})^{c}$; for instance, if $X$ is a noetherian, separated scheme and $\mathscr{A}$ is the category of quasicoherent sheaves on $X$, we know that $\mathbf{D}(\mathscr{A})^{c}=\mathbf{D}^{\text {perf }}(\operatorname{coh} X)$, the category of perfect complexes. In this special case, $\mathbf{K}(\operatorname{Inj} \mathscr{A})^{c}=\mathbf{D}^{b}$ (noeth $\mathscr{A}$ ) comes down to $\mathbf{D}^{b}(\operatorname{coh} X)$, the bounded derived category of the coherent sheaves on $X$. The general theory gives us the sequence of functors

$$
\mathbf{D}^{\text {perf }}(\operatorname{coh} X) \stackrel{Q_{\lambda}}{\longrightarrow} \mathbf{D}^{b}(\operatorname{coh} X) \stackrel{J_{\lambda}}{\longrightarrow} \mathbf{K}_{\mathrm{ac}}(\operatorname{Inj} \mathscr{A})^{c},
$$

and furthermore, it informs us that this sequence identifies the category $\mathbf{K}_{\mathrm{ac}}(\operatorname{Inj} \mathscr{A})^{c}$ of compact objects in $\mathbf{K}_{\mathrm{ac}}(\operatorname{Inj} \mathscr{A})$ with the idempotent completion of

$$
\mathbf{D}^{b}(\operatorname{coh} X) / \mathbf{D}^{\text {perf }}(\operatorname{coh} X)=\mathbf{D}_{\text {sing }}(X),
$$

the singularity category of $X$.

Jørgensen [2005] studied the analogue where injectives are replaced by projectives. Of course, Grothendieck abelian categories do not in general have enough projectives, so he restricted himself to the case where $\mathscr{A}$ is the category of modules over some ring. Under suitable noetherian hypotheses, he proved an analogue of Krause's 
theorem: the homotopy category $\mathbf{K}(R$-Proj $)$ is compactly generated, but strangely enough, the subcategory $\mathbf{K}(R \text {-Proj })^{c}$ of compact objects in $\mathbf{K}(R$-Proj) is naturally isomorphic to $\mathbf{D}^{b}\left(R^{\mathrm{op}} \text {-mod }\right)^{\text {op }}$, the opposite category of the bounded derived category of finitely presented $R^{\mathrm{op}}$-modules. Krause's theorem, in the special case where $\mathscr{A}$ is the category of $R$-modules, tells us that the subcategory $\mathbf{K}(R \text {-Inj })^{c}$ of compact objects in $\mathbf{K}\left(R\right.$-Inj) is naturally identified with $\mathbf{D}^{b}(R$-mod). If $R$ is a noetherian commutative ring, then both $\mathbf{K}(R$-Proj $)$ and $\mathbf{K}(R$-Inj $)$ are compactly generated, but the subcategories of compact objects are naturally the opposite of each other.

Iyengar and Krause [2006] studied this further and proved, among other things, that in the presence of a dualizing complex the categories $\mathbf{K}(R$-Proj) and $\mathbf{K}(R$-Inj) are equivalent. More precisely, tensoring with the dualizing complex induces an equivalence. Of course, it must also induce an equivalence on the subcategories of compact objects; that is, it must induce an equivalence

$$
\mathbf{D}^{b}\left(R^{\mathrm{op}}-\mathrm{mod}\right)^{\mathrm{op}} \rightarrow \mathbf{D}^{b}(R \text {-mod }) .
$$

This equivalence turns out to be the usual one of Grothendieck duality.

The results raise the obvious question: what is the right generality in which the results hold? Since Grothendieck abelian categories rarely have enough projectives, Jørgensen's results all assumed that he was working over a ring; in other words, they were restricted to the affine case of Grothendieck duality. In [Neeman 2008; 2010], I studied this problem and proved several improvements of Jørgensen's results, and Murfet [2007] carried the project further in his $\mathrm{PhD}$ thesis. One striking feature of my results was that much of what Jørgensen proved for $\mathbf{K}(R$-Proj) was true without the noetherian hypothesis, which raises the question: to what extent is the noetherian hypothesis necessary in Krause's results? On the face of it, the situation looks hopeless unless we assume that the category $\mathscr{A}$ is locally noetherian because without the noetherian hypothesis direct sums of injectives need not be injective. Hence, $\mathbf{K}(R$-Inj) does not obviously have coproducts in general, and without coproducts, one doesn't have a good notion of compact objects.

The first result of the current article, also found as Example 5 in [Krause 2012, pp. 778-779], addresses this:

Theorem 2.13 and Corollary 2.14. Let $\mathscr{A}$ be any Grothendieck abelian category not necessarily locally noetherian. Then the inclusion of $\mathbf{K}(\operatorname{Inj} \mathscr{A})$ into $\mathbf{K}(\mathscr{A})$ has a left adjoint I $: \mathbf{K}(\mathscr{A}) \rightarrow \mathbf{K}(\operatorname{Inj} \mathscr{A})$. It formally follows that $\mathbf{K}(\operatorname{Inj} \mathscr{A})$ has coproducts.

Remark. Krause's proof is based on the work of Bican, El Bashir, and Enochs [Bican et al. 2001], which means that it works far more generally than the argument we give here, but unfortunately, the adjoint is not very explicit. For the proof of the next theorem, we need to have a handle on this adjoint; hence, we give a different proof. 
While I do not understand the situation well enough to say when $\mathbf{K}(\operatorname{Inj} \mathscr{A})$ is compactly generated, I do have the following result:

Theorem 3.13. There is a regular cardinal $\mu$ for which the category $\mathbf{K}(\operatorname{Inj} \mathscr{A})$ is $\mu$-compactly generated (in the sense of [Neeman 2001, Definition 8.1.6] or [Krause 2001]).

In the algebrogeometric situation, it may well be that the categories $\mathbf{K}(\operatorname{Inj} X)$ and $K_{m}(\operatorname{Proj} X)$ can be equivalent even when $X$ is not noetherian. Here $K_{m}(\operatorname{Proj} X)$ is Murfet's mock homotopy category of projectives; for nonaffine schemes, this is the right generalization. Both $\mathbf{K}(\operatorname{Inj} X)$ and $K_{m}(\operatorname{Proj} X)$ have coproducts and are $\mu$-compactly generated for $\mu$ sufficiently large. In the noetherian case, they are equivalent whenever $X$ has a dualizing complex, and part of the interest of the results is that they might lead to a nonnoetherian generalization.

Recall that, if $\mathscr{A}$ is locally noetherian and $\mathbf{D}(\mathscr{A})$ is compactly generated, then the natural functors

$$
\mathbf{K}_{\mathrm{ac}}(\operatorname{Inj} \mathscr{A}) \stackrel{J}{\rightarrow} \mathbf{K}(\operatorname{Inj} \mathscr{A}) \stackrel{Q}{\rightarrow} \mathbf{D}(\mathscr{A})
$$

have right and left adjoints giving a recollement. It turns out that the right adjoints $J_{\rho}$ and $Q_{\rho}$ exist much more generally for any Grothendieck abelian category. But the left adjoints don't: we will produce an example of a locally noetherian Grothendieck abelian category $\mathscr{A}$ such that the functor $J$ does not respect products and hence cannot have a left adjoint. It will then follow, from [Krause 2005], that the category $\mathbf{D}(\mathscr{A})$ is not compactly generated.

The article is organized as follows. In Section 1, we recall that any Grothendieck abelian category is locally presentable, meaning there is a generator $g$ and a regular cardinal $\alpha$ so that $\operatorname{Hom}(g,-)$ commutes with $\alpha$-filtered colimits. We discuss this in some detail because we also want to prove that $\operatorname{Ext}^{1}(g,-)$ commutes with $\alpha$-filtered colimits.

In Section 2, we prove Theorem 2.13 and Corollary 2.14; for a given $X \in \mathbf{K}(\mathscr{A})$, we give an explicit construction of $I(X) \in \mathbf{K}(\operatorname{Inj} \mathscr{A})$ as a certain colimit. In Section 3 , we prove Theorem 3.13, showing the $\mu$-compact generation of $\mathbf{K}(\operatorname{Inj} \mathscr{A})$. The essence of the proof is to study the construction of $I(X)$ more carefully and see what it does to subobjects of $X$.

Finally, Section 4 contains the counterexample, the locally noetherian Grothendieck abelian category in which products of acyclic complexes of injectives need not be acyclic.

\section{Cardinality estimates in Grothendieck abelian categories}

Throughout the section, we will assume that $\mathscr{A}$ is a Grothendieck abelian category and $g \in \mathscr{A}$ is a fixed generator. 
Lemma 1.1. Let $Y$ be an object of $\mathscr{A}$. If the cardinality of $\operatorname{Hom}_{\mathscr{A}}(g, Y)$ is $\leq \alpha$, then $Y$ has no more than $2^{\alpha}$ subobjects.

Proof. We have a map

$$
\{\text { subobjects of } Y\} \stackrel{\Phi}{\longrightarrow} \text { \{subsets of } \operatorname{Hom}(g, Y)\},
$$

which takes a subobject $X \subset Y$ to the subset $\Phi(X)=\operatorname{Hom}(g, X) \subset \operatorname{Hom}(g, Y)$. The map $\Phi$ is injective because we can recover $X$ from $\Phi(X)$ : the fact that $g$ is a generator allows us to choose an epimorphism $\bigsqcup_{\Lambda} g \rightarrow X$. Then the factorization

$$
\coprod_{\Lambda} g \rightarrow \coprod_{\operatorname{Hom}(g, X)} g \rightarrow X
$$

tells us that $X$ is the image in $Y$ of the natural map $\bigsqcup_{\operatorname{Hom}(g, X)} g \rightarrow Y$.

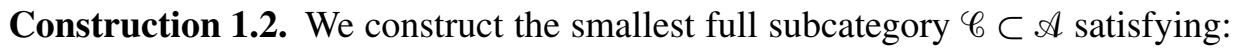

(i) The generator $g$ belongs to $\mathscr{b}$.

(ii) If $X$ is an object of $\mathscr{C}$, then so are all the subquotients of $g^{\# \operatorname{Hom}(g, X)}$. Here \# $\operatorname{Hom}(g, X)$ stands for the cardinality of $\operatorname{Hom}(g, X)$, and for a cardinal $\alpha$, we let $g^{\alpha}$ be the coproduct of $\alpha$ copies of $g$.

Lemma 1.3. There is only a set of isomorphism classes of objects of $\mathscr{C}$.

Proof. We build up $\mathscr{C}$ in countably many steps: we start with $\mathscr{C}_{0}=\{g\}$ and then construct $\mathscr{C}_{n+1}$ out of $\mathscr{C}_{n}$ by throwing in all the subquotients of $g^{\# \operatorname{Hom}(g, X)}$ for all $X \in \mathscr{C}_{n}$. We let $\mathscr{C}$ be the union of the $\mathscr{C}_{n}$.

Definition 1.4. We let $\alpha>\aleph_{0}$ be a regular cardinal such that (i) $2^{\# \operatorname{Hom}(g, Y)}<\alpha$ for all $Y \in \mathscr{C}$, and (ii) there are $<\alpha$ isomorphism classes of objects in $\mathscr{C}$.

Lemma 1.5. Let $Z$ be an object of $\mathscr{C}$ and $f: Z^{\prime} \rightarrow Z$ an epimorphism in $A$. Then there is an object $Y \in \mathscr{C}$ and a morphism $g: Y \rightarrow Z^{\prime}$ so that the composite $Y \rightarrow Z^{\prime} \rightarrow Z$ is epi.

Proof. We may choose an epimorphism $\bigsqcup_{\Lambda} g \rightarrow Z^{\prime}$ and consider the composite epimorphism

$$
\coprod_{\Lambda} g \rightarrow Z^{\prime} \rightarrow Z
$$

Let $M$ be the image of $\Lambda$ in $\operatorname{Hom}(g, Z)$; we may choose a splitting to the surjection $\Lambda \rightarrow M$. The composite

$$
\coprod_{M} g \rightarrow \coprod_{\Lambda} g \rightarrow Z^{\prime} \rightarrow Z
$$

is an epimorphism from a subquotient of $g^{\# \operatorname{Hom}(g, Z)}$ to $Z$ and factors through $Z^{\prime}$. 
Lemma 1.6. Let $\Phi$ be a $\beta$-filtered category for some regular cardinal $\beta$, let $F$ : $\Phi \rightarrow A$ be a functor, and let $\varphi: F \rightarrow Z$ be a natural transformation from $F$ to the constant functor that takes every $i \in \mathscr{I}$ to the object $Z \in \mathcal{A}$ and every morphism to the identity. Assume that the map $\operatorname{colim} F \rightarrow Z$ is epi and that $Z$ has fewer than $\beta$ subobjects. Then there exists some object $i \in \mathscr{I}$ with $\mathrm{Fi} \rightarrow Z$ epi.

Proof. Consider the set $S$ of subobjects of $Z$ that are images of $F i \rightarrow Z$ for some $i \in \mathscr{I}$. For each $X \in S$, choose an object $\rho(X) \in \mathscr{I}$ so that the image of $F(\rho(X)) \rightarrow Z$ is $X$. There are fewer than $\beta$ such $\rho(X)$, and hence, we may choose an object $j \in \mathscr{I}$ and, for each $X$, a morphism $\rho(X) \rightarrow j$. Then $\operatorname{Im}(F j \rightarrow Z)$ belongs to $S$ and contains all the other elements of $S$ as subobjects. The epimorphism colim $F i \rightarrow Z$ factors through $\operatorname{Im}(F j \rightarrow Z)$, and hence, $F j \rightarrow Z$ is epi.

Lemma 1.7. Every object of $\mathscr{C}$ is $\alpha$-presentable in $A$.

Proof. Let $Z$ be an object of $\mathscr{b}$; we need to show that $\operatorname{Hom}(Z,-)$ commutes with $\alpha$-filtered colimits in $\mathscr{A}$. Let $\mathscr{I}$ be an $\alpha$-filtered small category, and let $F: \mathscr{I} \rightarrow \mathscr{A}$ be a functor. We need to show that the natural map

$$
\Phi:{\underset{\operatorname{colim}}{\longrightarrow}}_{i \in \Phi} \operatorname{Hom}(Z, F i) \rightarrow \operatorname{Hom}\left(Z, \stackrel{\operatorname{colim}}{\longrightarrow}_{i \in \mathscr{\Phi}} F i\right)
$$

is an isomorphism. We will prove that $\Phi$ is surjective and injective.

Let us prove the injectivity of $\Phi$ first. An element in the kernel of $\Phi$ may be represented by a morphism $\theta: Z \rightarrow F i$ so that the composite

$$
Z \rightarrow F i \rightarrow \underset{\operatorname{colim}_{i \in I}}{\mathrm{Fi}} i
$$

vanishes. We need to show that for some $\rho: i \rightarrow j$ the composite $Z \rightarrow F i \stackrel{F \rho}{\longrightarrow} F j$ vanishes. Let $i / \mathscr{I}$ be the category whose objects are maps $\rho: i \rightarrow j$ in $\mathscr{I}$ and whose morphisms are commutative triangles

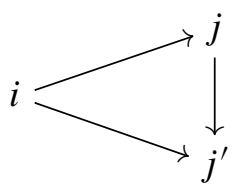

We have an exact sequence of functors on $i / \mathscr{I}$ that takes each object $\rho: i \rightarrow j$ to

$$
0 \rightarrow \operatorname{Ker}(Z \rightarrow F j) \rightarrow Z \rightarrow F j
$$

The category $i / \mathscr{I}$ is filtered, and hence, the colimit in the Grothendieck category $\mathscr{A}$ is the exact sequence

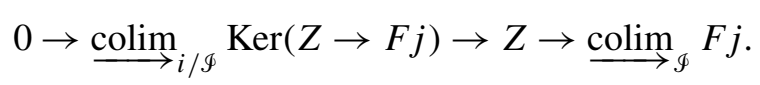

The fact that the map $Z \rightarrow \underset{\text { colim }_{g}}{\longrightarrow} F j$ vanishes means that $Z$ must be the colimit

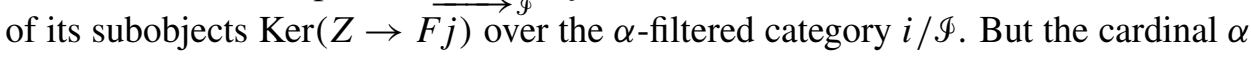


was chosen to be larger than $2^{\# \operatorname{Hom}(g, Z)}$, and Lemma 1.1 tells us that $Z \in \mathscr{C}$ has fewer than $\alpha$ subobjects. By Lemma 1.6, there is some object $\rho: i \rightarrow j$ in $\mathscr{I}$ with $\operatorname{Ker}(Z \rightarrow F j)=Z$.

Next we prove the surjectivity of $\Phi$. Let $L={\underset{\text { colim }}{\longrightarrow} i \in I}_{i} F i$; suppose we take an element of $\operatorname{Hom}(Z, L)$, that is, a map $\varphi: Z \rightarrow L$. For each $i$, we form the pullback square

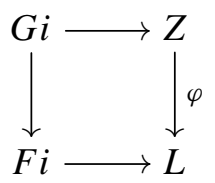

Then the $G i$ extend to a functor $G: \mathscr{I} \rightarrow \mathscr{A}$. Taking the colimit over the filtered category $\mathscr{I}$, we obtain a pullback square

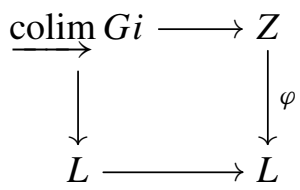

from which we conclude that the map colim $G i \rightarrow Z$ is epi (actually, it's even an isomorphism). But $\Phi$ is $\alpha$-filtered, and Lemma 1.1 tells us that $Z \in \mathscr{C}$ has fewer than $\alpha$ subobjects. By Lemma 1.6, there is an object $i \in \mathscr{I}$ so that $G i \rightarrow Z$ is epi.

By Lemma 1.5, we may choose an object $Y \in \mathscr{C}$ and a morphism $Y \rightarrow G i$ so that the composite $Y \rightarrow G i \rightarrow Z$ is epi. Let $X$ be the kernel of the epimorphism $Y \rightarrow Z$; because $X$ is a subobject of $Y \in \mathscr{C}$, it lies in $\mathscr{C}$ and the composite $X \rightarrow F i \rightarrow L$ vanishes. By the injectivity of $\Phi$, there must be some $\rho: i \rightarrow j$ in $\mathscr{I}$ so that the composite $X \rightarrow F i \stackrel{F(\rho)}{\longrightarrow} F j$ vanishes. But the vanishing of $X \rightarrow Y \rightarrow F i \rightarrow F j$ means that the map $Y \rightarrow F j$ factors through $Y \rightarrow Z \rightarrow F j$. We have found a $Z \rightarrow F j$ that maps under $\Phi$ to $\varphi: Z \rightarrow L=\stackrel{\operatorname{colim}}{\longrightarrow} i \in I_{F i}$.

Lemma 1.8. Let $X$ be an object of $\mathscr{C}$ and $Z$ an object of $A$. For every element $z \in \operatorname{Ext}_{\mathscr{A}}^{n}(X, Z)$, there exists an object $Y \in \mathscr{C}$, a morphism $Y \rightarrow Z$, and an element $y \in \operatorname{Ext}_{\mathscr{C}}^{n}(X, Y)$ so that $y$ maps to $z$ under the natural map.

Proof. The case $n=0$ is trivial; we may take $y$ to be the identity. Suppose therefore $n>0$; then $z$ is represented by an extension

$$
0 \rightarrow Z \rightarrow \cdots \rightarrow W \rightarrow X \rightarrow 0 .
$$

By Lemma 1.5, we may choose an object $W^{\prime} \in \mathscr{C}$ and a morphism $W^{\prime} \rightarrow W$ so that the composite $W^{\prime} \rightarrow W \rightarrow X$ is epi. If $X^{\prime}$ is the kernel of $W^{\prime} \rightarrow X$, then $X^{\prime}$ is a subobject of the object $W^{\prime} \in \mathscr{C}$ and hence belongs to $\mathscr{C}$. And the extension $z \in \operatorname{Ext}_{\mathscr{A}}^{n}(X, Z)$ is equivalent to the concatenation of $0 \rightarrow X^{\prime} \rightarrow W^{\prime} \rightarrow X \rightarrow 0$ in $\operatorname{Ext}_{\mathscr{C}}^{1}\left(X, X^{\prime}\right)$ with an extension $z^{\prime} \in \operatorname{Ext}_{\mathscr{A}}^{n-1}\left(X^{\prime}, Z\right)$. Induction on $n$ now gives the result. 
Corollary 1.9. The category $\mathscr{b} \subset A$ is closed under extensions.

Proof. Let $0 \rightarrow Z \rightarrow Y \rightarrow X \rightarrow 0$ be an extension with $X, Z \in \mathscr{C}$ and $Y \in \mathscr{A}$. By Lemma 1.8, there is an extension $0 \rightarrow A \rightarrow B \rightarrow X \rightarrow 0$ in $\mathscr{C}$ and a map $A \rightarrow Z$ connecting the extensions. Thus, $Y$ must be the pushout in the diagram

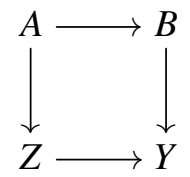

and hence, $Y$ is a quotient of $B \oplus Z \in \mathscr{C}$ and must belong to $\mathscr{C}$.

Lemma 1.10. For every object $X \in \mathscr{C}$ and every $n \geq 0$, the functor $\operatorname{Ext}^{n}(X,-)$ commutes with $\alpha$-filtered colimits.

Proof. The case $n=0$ was proved in Lemma 1.7, and we will now prove the general case. Let $\mathscr{I}$ be an $\alpha$-filtered category, and let $F: \mathscr{I} \rightarrow \mathscr{A}$ be a functor. We need to show that the natural map

$$
\Phi:{\underset{\operatorname{colim}}{\longrightarrow}}_{i \in \Phi} \operatorname{Ext}^{n}(X, F i) \rightarrow \operatorname{Ext}^{n}\left(X,{\underset{\operatorname{colim}}{\longrightarrow}}_{i \in \mathscr{I}} F i\right)
$$

is an isomorphism. We will prove that $\Phi$ is surjective and injective.

We prove surjectivity first. Given an element

$$
z \in \operatorname{Ext}^{n}\left(X,{\underset{\operatorname{colim}}{\longrightarrow}}_{i \in \mathscr{I}} F i\right),
$$

there is by Lemma 1.8 an object $Y \in \mathscr{C}$, an extension $y \in \operatorname{Ext}^{n}(X, Y)$, and a morphism $Y \rightarrow \stackrel{\text { colim }}{\longrightarrow}_{i \in \mathscr{I}} F i$ taking $y$ to $z$. By Lemma 1.7, the map from $Y \in \mathscr{C}$ to the $\alpha$-filtered colimit factors through some $F i$, and the surjectivity of $\Phi$ follows.

Next we prove injectivity by induction on $n$. Let

$$
L={\underset{\operatorname{colim}}{\longrightarrow}}_{i \in \mathscr{\Phi}} F i
$$

and suppose we are given a $\theta \in \operatorname{Ext}^{n}(X, F i)$ that maps to zero in $\operatorname{Ext}^{n}(X, L)$. In the proof of Lemma 1.8, we produced objects $W^{\prime}, X^{\prime} \in \mathscr{C}$, an exact sequence $0 \rightarrow X^{\prime} \rightarrow W^{\prime} \rightarrow X \rightarrow 0$ in $\mathscr{C}$, and an element $\theta^{\prime} \in \operatorname{Ext}^{n-1}\left(X^{\prime}, F i\right)$ mapping to $\theta \in \operatorname{Ext}^{n}(X, F i)$. In the commutative diagram with exact rows

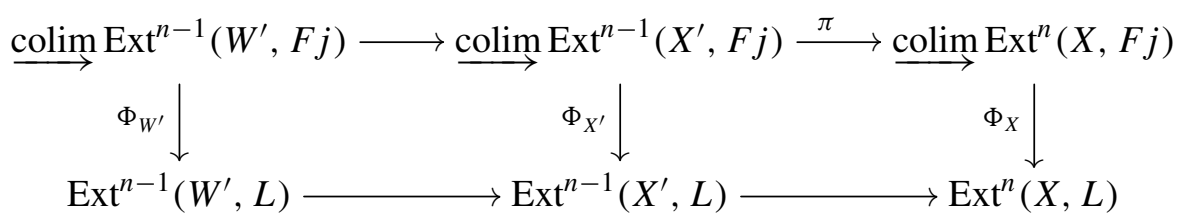

we have $\theta \in \underset{\operatorname{colim}}{\longrightarrow} \operatorname{Ext}^{n}(X, F j)$ and $\theta^{\prime} \in \underset{\operatorname{colim}}{\longrightarrow} \operatorname{Ext}^{n-1}\left(X^{\prime}, F j\right)$ satisfying $\pi\left(\theta^{\prime}\right)=\theta$ and $\Phi_{X}(\theta)=0$. By induction on $n$, we know that the maps $\Phi_{X^{\prime}}$ and $\Phi_{W^{\prime}}$ are isomorphisms. A short diagram chase establishes that $\theta$ vanishes as an element of $\stackrel{\operatorname{colim}}{\longrightarrow} \operatorname{Ext}^{n}(X, F j)$. 
Definition 1.11. Choose a regular cardinal $\mu$ so that:

(i) For each object $X \in \mathscr{C}$ and for each quotient $Y$ of $g^{\alpha}$, where $\alpha$ is as in Definition 1.4, we have \# $\operatorname{Hom}(X, Y)<\mu$.

(ii) The object $g^{\alpha}$ has $<\mu$ quotients.

(iii) The product of $\leq \alpha$ cardinals, all less than $\mu$, is less than $\mu$.

Remark 1.12. If we let $\beta$ be any cardinal $\geq \alpha$ satisfying parts (i) and (ii) of Definition 1.11, then the successor of $2^{\beta}$ satisfies all three hypotheses on $\mu$. The product of $\leq \alpha$ cardinals, all less than $\mu$, is the product of $\leq \alpha$ cardinals all $\leq 2^{\beta}$ and is bounded above by $\left(2^{\beta}\right)^{\alpha}=2^{\beta \times \alpha}=2^{\beta}$.

Definition 1.13. Let $\mu$ be as in Definition 1.11. We define $\mathscr{B} \subset \mathscr{A}$ to be the full subcategory of $\mathscr{A}$ consisting of the objects $Y$ with \# $\operatorname{Hom}(g, Y)<\mu$.

Lemma 1.14. An object $Y$ belongs to $\mathscr{B}$ if and only if it is the quotient to $g^{\lambda}$ for some $\lambda<\mu$. And if $Y$ belongs to $\mathscr{B}$, then $\# \operatorname{Hom}(X, Y)<\mu$ for all $X \in \mathscr{C}$.

Proof. Every $Y$ is the quotient of $g^{\# \operatorname{Hom}(g, Y)}$. If $Y$ happens to belong to $\mathscr{B}$, then $\lambda=\# \operatorname{Hom}(g, Y)<\mu$, so $Y$ is a quotient as specified. We need to prove the converse: any quotient of $g^{\lambda}, \lambda<\mu$, belongs to $\mathscr{B}$. Let $Y$ be such a quotient; to show that $Y$ belongs to $\mathscr{B}$, it suffices to prove that \# $\operatorname{Hom}(g, Y)<\mu$, but we actually want the refinement that \# $\operatorname{Hom}(X, Y)<\mu$ for every $X \in \mathscr{C}$.

Choose an epimorphism $g^{\lambda} \rightarrow Y$ and $X \in \mathscr{C}$. We note that $Y$ is the $\alpha$-filtered colimit of the subobjects $F i, i \in \mathscr{I}$, where $F i$ is the image in $Y$ of the map from a summand $g^{\beta_{i}} \subset g^{\lambda}$ with $\beta_{i}<\alpha$. By Lemma 1.7, every map $X \rightarrow Y$ factors as $X \rightarrow F i \rightarrow Y$ for some $i \in \mathscr{I}$. But \# $\operatorname{Hom}(X, F i)<\mu$ by Definition 1.11(i), and there are fewer than $\lambda^{\alpha}<\mu$ different inclusions $g^{\beta_{i}} \subset g^{\lambda}$. Therefore, there are fewer than $\mu$ factorizations $X \rightarrow F i \rightarrow Y$.

Proposition 1.15. The category $\mathscr{B}$ satisfies the following properties:

(i) The coproduct of $<\mu$ objects in $\mathscr{B}$ lies in $\mathscr{B}$.

(ii) Any subquotient of an object in $\mathscr{B}$ belongs to $\Re$.

(iii) Any extension of objects in $\mathscr{B}$ lies in $\mathscr{B}$.

(iv) $\mathscr{C}$ is contained in $\mathscr{B}$.

(v) For all $X \in \mathscr{C}$, all $Z \in \mathscr{B}$, and all integers $n \geq 0$, we have \# $\operatorname{Ext}^{n}(X, Z)<\mu$.

Proof. (i) Let $\left\{Y_{i} \mid i \in \mathscr{I}\right\}$ be a set of $<\mu$ objects in $\mathscr{B}$. Each $Y_{i}$ is the quotient of $g^{\# \operatorname{Hom}\left(g, Y_{i}\right)}$, and hence, the coproduct of the $Y_{i}$ is a quotient of $g^{\sum_{i} \# \operatorname{Hom}\left(g, Y_{i}\right)}$. And $\sum_{i} \# \operatorname{Hom}\left(g, Y_{i}\right)$ is the sum of fewer than $\mu$ cardinals, each $<\mu$, and hence is $<\mu$. (ii) If $Y$ belongs to $\mathscr{B}$, it is a quotient of $g^{\lambda}, \lambda<\mu$, and hence, so is any of its quotients. Also \# $\operatorname{Hom}(g, Y)<\mu$, and for any subobject $X$, we have \# $\operatorname{Hom}(g, X) \leq$ $\# \operatorname{Hom}(g, Y)<\mu$. 
(iii) Given an extension $0 \rightarrow X \rightarrow Y \rightarrow Z \rightarrow 0$, we have an exact sequence $0 \rightarrow \operatorname{Hom}(g, X) \rightarrow \operatorname{Hom}(g, Y) \rightarrow \operatorname{Hom}(g, Z)$. If $X$ and $Z$ belong to $\mathscr{B}$, then \# $\operatorname{Hom}(g, X)<\mu$ and \# $\operatorname{Hom}(g, Z)<\mu$, and \#Hom $(g, Y)$ is the sum of fewer than $\mu$ cardinals all smaller than $\mu$.

(iv) Suppose $Y \in \mathscr{C}$. By the definition of $\alpha$, we have $\# \operatorname{Hom}(g, Y)<\alpha$, and $\mu$ was chosen larger than $\alpha$.

(v) There are fewer than $\alpha$ objects in $\mathscr{C}$, and for any pair of objects $X, Y \in \mathscr{C}$, we have that \# $\operatorname{Hom}(X, Y)<\mu$. Hence, there are fewer than $\mu$ sequences in $\mathscr{C}$ of length $n$

$$
0 \rightarrow Y \rightarrow W_{1} \rightarrow \cdots \rightarrow W_{n} \rightarrow X \rightarrow 0 .
$$

Some of these sequences will be exact, and up to equivalence, they define fewer than $\mu$ elements in groups $\operatorname{Ext}_{\mathscr{C}}^{n}(X, Y)$. By Lemma 1.8, we have that any element of $\operatorname{Ext}^{n}(X, Z)$ is the image under some map in $\operatorname{Hom}(Y, Z)$ of an element in $\operatorname{Ext}_{\mathscr{C}}^{n}(X, Y)$. $\operatorname{But} \# \operatorname{Hom}(Y, Z)<\mu$ by Lemma 1.14 ; hence, there are fewer than $\mu$ ways of picking a triple

$$
Y \in \mathscr{C}, \quad y \in \operatorname{Ext}_{\mathscr{C}}^{n}(X, Y), \quad \text { and } \quad f \in \operatorname{Hom}(Y, Z) .
$$

Remark 1.16. If $F: \mathscr{I} \rightarrow \mathscr{B}$ is a functor and $\mathscr{I}$ has fewer than $\mu$ objects, then the colimit of $F$ belongs to $\mathscr{B}$. This is because the colimit is a quotient of the coproduct of $F i$ over all objects $i \in \mathscr{I}$. The coproduct belongs to $\mathscr{B}$ by Proposition 1.15(ii) and its quotient the colimit by Proposition 1.15(iii).

Remark 1.17. Let $q$ be the coproduct of all the quotients of the generator $g$. Then for every $n \geq 0$ and any object $Z \in \mathscr{B}$, we have \# $\operatorname{Ext}^{n}(q, Z)<\mu$. The reason is the following. Write $q=\bigsqcup_{M} x_{m}$ to express $q$ as the coproduct of all the quotients $x_{m}$ of $g$. Then

$$
\operatorname{Ext}^{n}(q, Z)=\prod_{M} \operatorname{Ext}^{n}\left(x_{m}, Z\right)
$$

with each $x_{m} \in \mathscr{C}$ and $Z \in \mathscr{B}$. By Proposition 1.15(v), we have that \# $\operatorname{Ext}^{n}\left(x_{m}, Z\right)<\mu$ for each $x_{m}$, and there are fewer than $\alpha$ objects $x_{m} \in \mathscr{C}$. Definition 1.11(iii) guarantees that $\# \operatorname{Ext}^{n}(q, Z)<\mu$.

Proposition 1.18. The category $\mathscr{\Re}$ is precisely the full subcategory of $\mu$-presentable objects of $A$.

Proof. Let $Y$ be a $\mu$-presentable object in $\mathscr{A}$, and let $g^{\gamma} \rightarrow Y$ be an epimorphism. Then $Y$ is the $\mu$-filtered colimit of all the subobjects $\{F i \mid i \in \mathscr{Y}\}$, where $F i$ is the image in $Y$ of some summand $g^{\lambda_{i}} \subset g^{\gamma}$ with $\lambda_{i}<\mu$. The identity map $Y \rightarrow Y$ is a map from the $\mu$-presentable object $Y$ to the $\mu$-filtered colimit $Y=\underset{\text { colim }}{\longrightarrow} F i$ and hence must factor through some $F i$. But then the map $g^{\lambda_{i}} \rightarrow F i \rightarrow \overrightarrow{Y \text { is }}$ the composite of two epimorphisms and is epi, and $\lambda_{i}<\mu$. Thus, $Y \in \mathscr{B}$. 
Next we must prove that every $Y \in \mathscr{B}$ is $\mu$-presentable. Choose an epimorphism $g^{\lambda} \rightarrow Y$ with $\lambda<\mu$; its kernel $K$ is a subobject of a coproduct of fewer than $\mu$ copies of $g \in \mathscr{B}$ and hence belongs to $\mathscr{B}$. Thus, we may choose an epimorphism $g^{\nu} \rightarrow K$ with $v<\mu$. We therefore have a presentation of $Y$

$$
g^{\nu} \rightarrow g^{\lambda} \rightarrow Y \rightarrow 0
$$

with $\lambda, v<\mu$. But $g$ is $\alpha$-presentable by Lemma 1.7 and hence also $\mu$-presentable for our choice of $\mu>\alpha$. The $\mu$-presentability of $Y$ follows from the presentation.

An immediate corollary is:

Corollary 1.19. Any Grothendieck abelian category is locally presentable (in the sense of [Gabriel and Ulmer 1971]).

Definition 1.20. Let $v$ be an infinite cardinal. We define $\mathscr{A}^{v} \subset \mathscr{A}$ to be the full subcategory of all $v$-presentable objects.

Remark 1.21. In Definition 1.11, we chose a regular cardinal $\mu$, and in Proposition 1.18 , we saw that $\mathscr{A}^{\mu}=\mathscr{B}$ with $\mathscr{B}$ as in Definition 1.13. As it happens, we chose $\mu$ sufficiently large so that $\mathscr{A}^{\mu}$ satisfies all the nice properties of Lemma 1.14, Proposition 1.15, and Remark 1.16.

\section{An adjoint to the inclusion $K(\operatorname{Inj} \mathscr{A}) \hookrightarrow K(\mathscr{A})$}

Let $\mathscr{A}$ be a Grothendieck abelian category, let $\mathbf{K}(\mathscr{A})$ be the homotopy category of chain complexes in $\mathscr{A}$, and let $\mathbf{K}(\operatorname{Inj} \mathscr{A})$ be the full subcategory whose objects are the chain complexes of injectives. There is an obvious inclusion $\mathbf{K}(\operatorname{Inj} \mathscr{A}) \hookrightarrow \mathbf{K}(\mathscr{A})$, and in this section, we will study its left adjoint.

We begin with some preliminaries.

Lemma 2.1. Every bounded-below, acyclic complex lies in ${ }^{\perp} \mathbf{K}(\operatorname{Inj} \mathscr{A})$. We remind the reader: this means that any chain map $A \rightarrow E$ from a bounded-below, acyclic complex to a complex of injectives is null-homotopic.

Proof. If $A$ vanishes in degrees $<n$, then the map $A \rightarrow E$ factors through the brutal truncation $\beta^{\geq n} E$; the factorization is the obvious

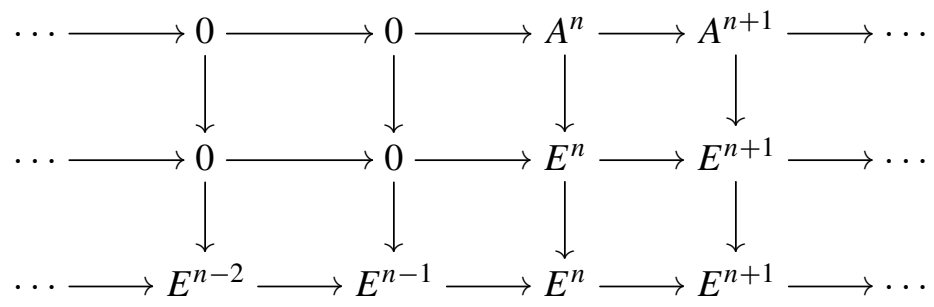

The map $A \rightarrow \beta^{\geq n} E$ is a chain map from an acyclic complex to a bounded-below complex of injectives and hence null-homotopic. 
The converse is not true: objects of ${ }^{\perp} \mathbf{K}(\operatorname{Inj} \mathscr{A})$ do not have to be bounded below. But they do have to be acyclic.

Lemma 2.2. Every object of ${ }^{\perp} \mathbf{K}(\operatorname{Inj} \mathscr{A})$ is acyclic.

Proof. Let $E$ be an injective cogenerator of the abelian category $\mathscr{A}$. Then $\operatorname{Hom}(X$, $\left.\Sigma^{-n} E\right)=0$ if and only if $H^{n}(X)=0$.

Lemma 2.3. Let $f: X \rightarrow Y$ be quasi-isomorphism of chain complexes, and assume $f^{i}: X^{i} \rightarrow Y^{i}$ is an isomorphism for all $i \ll 0$. Then, for any chain complex of injectives $E$, the natural map

$$
\operatorname{Hom}_{\mathbf{K}(\mathscr{A})}(Y, E) \stackrel{\operatorname{Hom}(f, E)}{\longrightarrow} \operatorname{Hom}_{\mathbf{K}(\mathscr{A})}(X, E)
$$

is an isomorphism.

Proof. In the triangle $A \rightarrow X \stackrel{f}{\rightarrow} Y \rightarrow \Sigma A$, we have that $f$ is a quasi-isomorphism and hence $A$ is acyclic. Furthermore, the fact that $f^{i}: X^{i} \rightarrow Y^{i}$ is an isomorphism for $i \ll 0$ means that $A$ is homotopy-equivalent to a bounded-below complex, and Lemma 2.1 tells us that $A \in{ }^{\perp} \mathbf{K}(\operatorname{Inj} \mathscr{A})$. The result now follows by applying $\operatorname{Hom}(-, E)$ to the triangle.

Remark 2.4. In Lemma 2.3, we saw that any chain map $X \rightarrow E$ factors up to homotopy through $X \rightarrow Y$. We wish to consider the factorizations not only up to homotopy, and hence, we will work in $\mathbf{C}(\mathscr{A})$, the category of chain complexes in $\mathscr{A}$ where the morphisms are genuine chain maps, not homotopy equivalence classes.

Lemma 2.5. Let $f: X \rightarrow Y$ be a morphism in $\mathbf{C}(\mathscr{A})$ whose mapping cone lies ${ }_{\text {in }}{ }^{\perp} \mathbf{K}(\operatorname{Inj} \mathscr{A})$. Suppose further that in each degree $i$ the map $f^{i}: X^{i} \rightarrow Y^{i}$ is a monomorphism. Let $E$ be a complex of injectives; then the map $\operatorname{Hom}(f, E)$ : $\operatorname{Hom}_{\mathbf{C}(\mathscr{A})}(Y, E) \rightarrow \operatorname{Hom}_{\mathbf{C}(\mathscr{A})}(X, E)$ is surjective. In other words, every chain map $X \rightarrow$ E factors through $f: X \rightarrow Y$, not only up to homotopy but in the category $\mathbf{C}(\mathscr{A})$.

Proof. Suppose we are given a chain map $h: X \rightarrow E$. By Lemma 2.3, it factors up to homotopy through $f: X \rightarrow Y$, meaning there exists a $g: Y \rightarrow E$ so that $h$ is homotopic to $g f$. Let $\Theta: X \Rightarrow E$ be a homotopy that works; then for every $i \in \mathbb{Z}$, we have a map $\Theta^{i}: X^{i} \rightarrow E^{i-1}$ with

$$
h-g f=\Theta \partial+\partial \Theta .
$$

But now $\Theta^{i}: X^{i} \rightarrow E^{i-1}$ is a morphism in $\mathscr{A}$ from $X^{i}$ to the injective object $E^{i-1}$, and we may therefore factor it through the monomorphism $f^{i}: X^{i} \rightarrow Y^{i}$. Thus, we construct maps $\Phi^{i}: Y^{i} \rightarrow E^{i-1}$ with $\Phi^{i} f^{i}=\Theta^{i}$. If we let

$$
g^{\prime}=g+\Phi \partial+\partial \Phi,
$$

then $h=g^{\prime} f$. 
In Lemma 2.5, we showed the existence of a factorization $h=g f$. Next we worry about uniqueness.

Lemma 2.6. Suppose $f: X \rightarrow Y$ and $h: X \rightarrow E$ are as in Lemma 2.5. Assume $g, g^{\prime}: \rightarrow E$ are two morphisms with $g f$ and $g^{\prime} f$ homotopic to $h$, and let $\Theta: X \Rightarrow E$ be a homotopy with

$$
g f-g^{\prime} f=\Theta \partial+\partial \Theta .
$$

Then there exists a homotopy $\Phi: Y \Rightarrow E$ with $\Phi f=\Theta$ and so that

$$
g-g^{\prime}=\Phi \partial+\partial \Phi .
$$

Proof. Note that Lemma 2.3 guarantees that $g$ is homotopic to $g^{\prime}$; the content of what we are about to prove is that the homotopy connecting them may be chosen to lift any given homotopy of $g f$ with $g^{\prime} f$.

Let us therefore choose any homotopy $\Phi^{\prime}$ connecting $g$ with $g^{\prime}$. Then $\Phi^{\prime} f$ is a homotopy connecting $g f$ with $g^{\prime} f$ as is $\Theta$; it follows that $\Theta-\Phi^{\prime} f$ is a chain map $X \rightarrow \Sigma E$. By Lemma 2.5, it has a factorization $\Theta-\Phi^{\prime} f=\rho f$ with $\rho: Y \rightarrow \Sigma E$ a chain map. But then $\Phi=\Phi^{\prime}+\rho$ is a homotopy of $g$ with $g^{\prime}$, and $\Theta=\Phi f$.

Definition 2.7. Let $\lambda$ be an ordinal and $\mathscr{K}$ a category. A sequence of length $\lambda$ in $\mathscr{K}$ is the following data:

(i) for every ordinal $i \leq \lambda$ an object $X_{i} \in \mathscr{K}$ and

(ii) for every pair of ordinals $i$ and $j$ with $i<j \leq \lambda$ a morphism $f_{i j}: X_{i} \rightarrow X_{j}$.

(iii) If $i<j<k \leq \lambda$, then the composite $X_{i} \stackrel{f_{i j}}{\longrightarrow} X_{j} \stackrel{f_{j k}}{\longrightarrow} X_{k}$ agrees with $f_{i k}$ : $X_{i} \rightarrow X_{k}$.

Lemma 2.8. Suppose $X$ is a sequence of length $\lambda$ in $\mathbf{C}(\mathscr{A})$, and assume that for every limit ordinal $j$ we have

$$
X_{j}=\underset{\text { colim}}{\longrightarrow} X_{i} .
$$

Suppose further that the mapping cone on every $X_{i} \rightarrow X_{i+1}$ belongs to ${ }^{\perp} \mathbf{K}(\operatorname{Inj} \mathscr{A})$ and that each of the maps $X_{i} \rightarrow X_{i+1}$ is a degreewise monomorphism. Then the mapping cones of all $f_{i j}: X_{i} \rightarrow X_{j}$ belong to ${ }^{\perp} \mathbf{K}(\operatorname{Inj} \mathscr{A})$.

Proof. We prove, by induction on $k \leq \lambda$, that the statement is true for all $f_{i j}$ with $i \leq j \leq k$. If $k=0$, there is nothing to prove.

Suppose the statement is true for $k$; we wish to prove it for $k+1$. Choose any $i<j \leq k+1$. If $i<j \leq k$, then the mapping cone on $f_{i j}$ lies in ${ }^{\perp} \mathbf{K}(\operatorname{Inj} \mathscr{A})$ by the inductive hypothesis. If $j=k+1$, then $i \leq k$ and $f_{i j}$ can be written as the composite $f_{k, k+1} f_{i k}$. Since the mapping cones on $f_{i k}$ and on $f_{k, k+1}$ both lie in ${ }^{\perp} \mathbf{K}(\operatorname{Inj} \mathscr{A})$, so does the mapping cone on the composite $f_{i j}=f_{k, k+1} f_{i k}$. 
Next suppose $k$ is a limit ordinal and the mapping cone on $f_{i j}$ lies in ${ }^{\perp} \mathbf{K}(\operatorname{Inj} \mathscr{A})$ for all $i<j<k$. We need to show that the mapping cone on $f_{i k} \operatorname{lies} \operatorname{in}{ }^{\perp} \mathbf{K}(\operatorname{Inj} \mathscr{A})$ for every $i<k$. Equivalently, we must prove that the induced map

$$
\operatorname{Hom}(f, E): \operatorname{Hom}_{\mathbf{K}(\mathscr{A})}\left(X_{k}, E\right) \rightarrow \operatorname{Hom}_{\mathbf{K}(\mathscr{A})}\left(X_{i}, E\right)
$$

is an isomorphism for every $E \in \mathbf{K}(\operatorname{Inj} \mathscr{A})$. Let us first prove the surjectivity.

Suppose we are given a chain map $h_{i}: X_{i} \rightarrow E$. By induction on $j$, we will factor $h_{i}$ in $\mathbf{C}(\mathscr{A})$ as $X_{i} \stackrel{f_{i j}}{\longrightarrow} X_{j} \stackrel{h_{j}}{\longrightarrow} E$. If we have produced the factorization through $h_{j}$, then Lemma 2.5 permits us to factor $h_{j}: X_{j} \rightarrow E$ as $X_{j} \stackrel{f_{j, j+1}}{\longrightarrow} X_{j+1} \stackrel{h_{j+1}}{\longrightarrow} E$. For limit ordinals $v$, we use the fact that $X_{v}=\underline{\text { colim }}_{i<v} X_{i}$ to extend the factorization. This finishes the induction, and we have a factorization of $h_{i}$ as $X_{i} \stackrel{f_{i k}}{\longrightarrow} X_{k} \stackrel{h_{k}}{\longrightarrow} E$. This factorization is in the category $\mathbf{C}(\mathscr{A})$, which is more than we need. It certainly reduces to a factorization in $\mathbf{K}(\mathscr{A})$.

Now we prove injectivity. Suppose we are given a chain map $h_{i}: X_{i} \rightarrow E$; we wish to prove that the factorization through $f_{i k}: X_{i} \rightarrow X_{k}$ is unique in $\mathbf{K}(\mathscr{A})$. Choose an $h_{k}: X_{k} \rightarrow E$ where the identity $h_{k} f_{i k}=h_{i}$ holds in $\mathbf{C}(\mathscr{A})$; the existence of such an $h_{k}$ has just been proved. Now take any $h: X_{k} \rightarrow E$ with $h f_{i k} \cong h_{i}$, that is, with $h f_{i k}$ homotopic to $h_{i}=h_{k} f_{i k}$; we need to prove that $h$ is homotopic to $h_{k}$. The proof is by choosing a homotopy $\Theta_{i}$ connecting $h f_{i k}$ with $h_{k} f_{i k}$ and then by induction on $j$ lifting it to a homotopy connecting $h f_{j k}$ with $h_{k} f_{j k}$ with $i \leq j \leq k$ using Lemma 2.6.

We will construct sequences to which we will apply Lemma 2.8. The maps $X_{i} \rightarrow X_{i+1}$ from which these sequences are built up will be obtained as follows.

Construction 2.9. Given an object $X \in \mathbf{C}(\mathscr{A})$, an integer $n$, and a monomorphism $X^{n} \rightarrow A$ in $A$, we form a map of chain complexes $f: X \rightarrow Y=B\left(X, n, X^{n} \rightarrow A\right)$ as follows:

(i) $f^{i}: X^{i} \rightarrow Y^{i}$ is the identity map $1: X^{i} \rightarrow X^{i}$ for all $i \neq n, n+1$.

(ii) In degrees $n$ and $n+1$, the commutative square

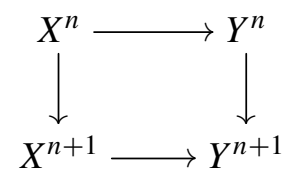

is just the pushout square

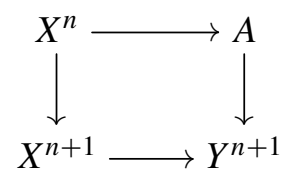

We could specify $A$, up to noncanonical isomorphism, by giving its class as an extension in $\operatorname{Ext}^{1}\left(A / X^{n}, X^{n}\right)$. In our applications, $A / X^{n}$ will be a large coproduct 
$A / X^{n}=q^{\beta}$ of $\beta$ copies of the object $q$ of Remark 1.17, and hence, it will suffice to give a subset $\Lambda \subset \operatorname{Ext}^{1}\left(q, X^{n}\right)$ of cardinality $\beta$. We will let $B(X, \Lambda)$ denote the corresponding complex $Y$.

Remark 2.10. Suppose we are given an integer $n$ and an object $y \in \mathscr{A}$. The trivial complex $T(y)$ is just the complex

$$
\cdots \rightarrow 0 \rightarrow 0 \rightarrow y \stackrel{1}{\rightarrow} y \rightarrow 0 \rightarrow 0 \rightarrow \cdots
$$

with the nonzero terms in degrees $n$ and $n+1$. Assume now that we are given an object $X \in \mathbf{C}(\mathscr{A})$ and a monomorphism $X^{n} \rightarrow A$ in $\mathscr{A}$. The morphism $f: X \rightarrow Y=$ $B\left(X, n, X^{n} \rightarrow A\right)$ of Construction 2.9 fits in a short exact sequence of complexes

$$
0 \rightarrow X \stackrel{f}{\rightarrow} Y \rightarrow T\left(A / X^{n}\right) \rightarrow 0
$$

and it immediately follows that $f$ is a monomorphism and a quasi-isomorphism. But the mapping cone in $\mathbf{K}(\mathscr{A})$ on the map $f$ is homotopic to a bounded complex and belongs to ${ }^{\perp} \mathbf{K}(\operatorname{Inj} \mathscr{A})$ by Lemma 2.1. Thus, $f$ is a suitable building block for constructing chains of complexes as in Lemma 2.8 .

Construction 2.11. Let $X \in \mathbf{C}(\mathscr{A})$ be an object. Let $g$ be our chosen generator for the abelian category $\mathscr{A}$, and let $M$ be the set of all the quotients of $g$. In Remark 1.17, we defined $q$ to be the coproduct of them all.

For each subset $\Lambda \subset \operatorname{Ext}^{1}\left(q, X^{n}\right)$, we consider the map $X \rightarrow B(X, \Lambda)$ of Construction 2.9. In the special case where $\Lambda=\operatorname{Ext}^{1}\left(q, X^{n}\right)$ is maximal, we denote the map as $X \rightarrow B(X, n)$. In this case, we know that the functor $\operatorname{Ext}^{1}(x,-)$ annihilates the map $X^{n} \rightarrow B(X, n)^{n}$ whenever $x$ is a direct summand of $q$, in particular for all quotients $x$ of $g$.

Given $X \in \mathbf{C}(\mathscr{A})$, we inductively define a sequence of length $\omega$ in $\mathbf{C}(\mathscr{A})$. At each step, we let the map $X_{i} \rightarrow X_{i+1}$ be $X_{i} \rightarrow B\left(X_{i}, n\right)$ for some suitable $n$ depending on $i$. The precise recipe is:

(i) $X_{0}=X$, and $X_{0} \rightarrow X_{1}$ is the map $X \rightarrow B(X, 0)$.

(ii) For an integer $i>0$, we define $X_{2 i-1} \rightarrow X_{2 i}$ to be $X_{2 i-1} \rightarrow B\left(X_{2 i-1}, i\right)$ while $X_{2 i} \rightarrow X_{2 i+1}$ is set to be $X_{2 i} \rightarrow B\left(X_{2 i},-i\right)$.

(iii) $X_{\omega}=\underset{\operatorname{colim}}{\longrightarrow} X_{n}$.

Lemma 2.12. Define the map $f_{X}: X \rightarrow J(X)$ to be the morphism $X \rightarrow X_{\omega}$ of Construction 2.11. Then $f_{X}$ is a degreewise monomorphism and is annihilated degreewise by $\operatorname{Ext}^{1}(x,-)$ whenever $x$ is a quotient of $g$. Furthermore, the mapping cone of $f_{X}$ lies in ${ }^{\perp} \mathbf{K}(\operatorname{Inj} \mathscr{A})$. 
Proof. By construction, $f_{X}$ is the colimit of degreewise monomorphisms and hence a degreewise monomorphism. The fact that $f_{X}$ is annihilated by $\operatorname{Ext}^{1}(x,-)$ in every degree $n$ is true because, depending on whether $n$ is positive or negative, either the map $X_{2|n|-1} \rightarrow X_{2|n|}$ or the map $X_{2|n|} \rightarrow X_{2|n|+1}$ will induce zero in degree $n$ under the functors $\operatorname{Ext}^{1}(x,-)$. That the mapping cone lies in ${ }^{\perp} \mathbf{K}(\operatorname{Inj} \mathscr{A})$ comes from Lemma 2.8.

Theorem 2.13. The natural inclusion $\mathbf{K}(\operatorname{Inj} \mathscr{A}) \rightarrow \mathbf{K}(\mathscr{A})$ has a left adjoint $I$.

Proof. Let $X$ be an arbitrary object of $\mathbf{C}(\mathscr{A})$. By transfinite induction, we define a chain of complexes $J^{\lambda}(X)$ for every ordinal $\lambda$. The rule is:

(i) $J^{0}(X)=X$.

(ii) If $J^{\lambda}(X)$ has been defined, then the map $J^{\lambda}(X) \rightarrow J^{\lambda+1}(X)$ is just $J^{\lambda}(X) \rightarrow$ $J\left(J^{\lambda}(X)\right)$.

(iii) If $\lambda$ is a limit ordinal, then $J^{\lambda}(X)={\underset{\text { colim}}{\longrightarrow}}_{i<\lambda} J^{i}(X)$.

Let $\alpha$ be the regular cardinal of Definition 1.4. Now consider the triangle

$$
A(X) \rightarrow X \rightarrow J^{\alpha}(X) \rightarrow \Sigma A(X) .
$$

Lemma 2.8 tells us that $A(X)$ belongs to ${ }^{\perp} \mathbf{K}(\operatorname{Inj} \mathscr{A})$. I assert that $J^{\alpha}(X)$ belongs to $\mathbf{K}(\operatorname{Inj} \mathscr{A})$; from the triangle and [Neeman 2001, Theorem 9.1.13], we deduce the existence of the adjoint and note that the adjoint takes $X$ to $I(X)=J^{\alpha}(X)$.

It remains to prove the assertion: we must show that in each degree $n$ the object $J^{\alpha}(X)^{n} \in \mathscr{A}$ is injective. Since $\alpha$ is an $\alpha$-filtered colimit of the ordinals $\lambda<\alpha$, Lemma 1.10 tells us that, for each quotient $x$ of the generator $g$ of $\mathscr{A}$,

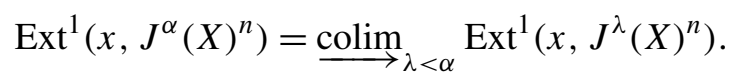

By construction, we know that the map

$$
\operatorname{Ext}^{1}\left(x, J^{\lambda}(X)^{n}\right) \rightarrow \operatorname{Ext}^{1}\left(x, J^{\lambda+1}(X)^{n}\right)
$$

is zero, and hence, the colimit vanishes. Thus, $\operatorname{Ext}^{1}\left(x, J^{\alpha}(X)^{n}\right)=0$ whenever $x$ is a quotient of the generator $g$, and hence, $J^{\alpha}(X)^{n}$ must be injective.

Corollary 2.14. The homotopy category $\mathbf{K}(\operatorname{Inj} \mathscr{A})$ satisfies TR5, meaning it has coproducts.

Proof. Given a collection of objects $\left\{X_{\lambda} \mid \lambda \in \Lambda\right\}$ in the category $\mathbf{K}(\operatorname{Inj} \mathscr{A})$, we can certainly form the coproduct in $\mathbf{K}(\mathscr{A})$; applying the functor $I$ to this coproduct gives the coproduct in $\mathbf{K}(\operatorname{Inj} \mathscr{A})$.

Remark 2.15. The construction of $I(X)$ out of $X$ was broken up into two steps. In the proof of Theorem 2.13, we constructed a sequence by letting $J^{i+1}(X)=$ $J\left(J^{i}(X)\right)$ for each ordinal $i$ and by taking colimits at limit ordinals. But this hides 
the fact that $J(Y)$ is constructed out of $Y$ as the colimit of a countable sequence where $Y_{i+1}=B\left(Y_{i}, n\right)$ for some suitable $n$ depending on $i$; see Construction 2.9. If we assemble it all into one long sequence, then we define a sequence where $X_{i+1}=B\left(X_{i}, n\right)$ for every ordinal $i$ but where the integer $n$ depends on the distance of the ordinal $i$ from its predecessor limit ordinal. And we recover the sequence $\left\{J^{i}(X)\right\}$ by restricting attention to $X_{i}$ for limit ordinals $i$.

\section{The $\mu$-compact generation of $K(\operatorname{Inj} A)$}

In Section 2, we proved Theorem 2.13: the inclusion $\mathbf{K}(\operatorname{Inj} \mathscr{A}) \rightarrow \mathbf{K}(\mathscr{A})$ has a left adjoint $I$. In the construction, we made many choices: even though we constructed a morphism $X \rightarrow I(X)=J^{\alpha}(X)$ in the category $\mathbf{C}(\mathscr{A})$, the construction is not functorial in $\mathbf{C}(\mathscr{A})$. The map sending $X$ to $I(X)$ becomes a well defined functor only in the homotopy category $\mathbf{K}(\mathscr{A})$, and $X \rightarrow I(X)$ is a natural transformation only at the homotopy level. Still $X$ can be expressed as the colimit of all its $\mu$-presentable subobjects with $\mu$ as in Definition 1.11, and we would like to express $I(X)$ as a $\mu$-filtered colimit.

Construction 3.1. Recall that $\mathscr{B} \subset \mathscr{A}$ was the category $\mathscr{A}^{\mu}$ of $\mu$-presentable objects in $\mathscr{A}$; see Proposition 1.18 and Definition 1.20. Let $X$ be an object in $\mathbf{C}(\mathscr{A})$, and let $\mathscr{I}$ be a full subcategory of subobjects $Y \subset X$ with $Y \in \mathbf{C}(\mathscr{B}) \subset \mathbf{C}(\mathscr{A})$. Assume $\mathscr{I}$ is $\mu$-filtered and its colimit is $X$. Construct the category $\mathscr{S}(\mathscr{I}, n)$ whose objects are subobjects $Y$ of $B(X, n)$ with the following properties:

(i) $Y \cap X$ belongs to $ף$.

(ii) The map $Y \cap X \rightarrow Y$ is an isomorphism in degrees $i \neq n, n+1$.

(iii) In degree $n$, we have a monomorphism $Y^{n} / Y^{n} \cap X^{n} \rightarrow B(X, n)^{n} / X^{n}$, and from the construction of $B(X, n)$, we know that $B(X, n)^{n} / X^{n}$ is the coproduct $q^{\beta}=$ $\bigsqcup_{\operatorname{Ext}^{1}\left(q, X^{n}\right)} q$ with $q$ as in Remark 1.17. We require that the monomorphism $Y^{n} / Y^{n} \cap X^{n} \rightarrow B(X, n)^{n} / X^{n}$ is the inclusion of a subcoproduct.

(iv) The square

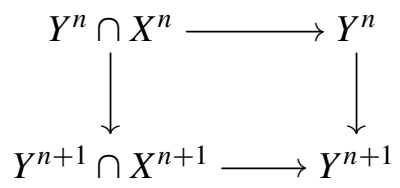

is a pushout.

Remark 3.2. Let us untangle what this means. In Construction $2.11, B(X, n)$ was defined so that there is a short exact sequence in $\mathbf{C}(\mathscr{A})$

$$
0 \rightarrow X \rightarrow B(X, n) \rightarrow T(q)^{\# \operatorname{Ext}\left(q, X^{n}\right)} \rightarrow 0
$$

where $T(q)$ is the trivial complex $\cdots \rightarrow 0 \rightarrow q \stackrel{1}{\rightarrow} q \rightarrow 0 \rightarrow \cdots$ concentrated in 
degrees $n$ and $n+1$. The conditions on the subobject $Y \subset B(X, n)$ that it must satisfy to belong to $\mathscr{E}(\mathscr{I}, n)$ come down to asking that $Y \cap X$ belongs to $\mathscr{I}$ and that in the map of short exact sequences

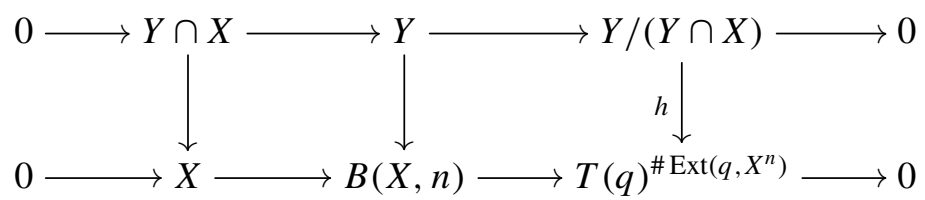

the monomorphism $h: Y /(Y \cap X) \rightarrow T(q)^{\# \operatorname{Ext}\left(q, X^{n}\right)}$ should be the inclusion of a subcoproduct. In degree $n$, we have a diagram

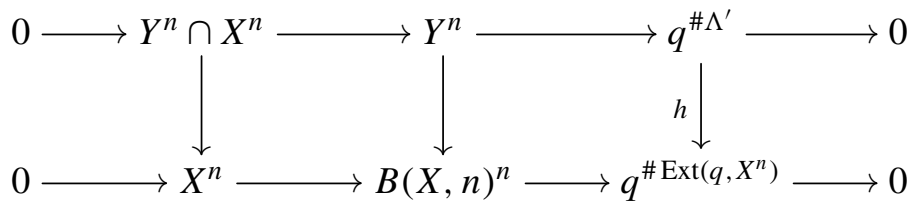

The top row of this diagram defines a map $\varphi: \Lambda^{\prime} \rightarrow \operatorname{Ext}^{1}\left(q, Y^{n} \cap X^{n}\right)$ giving the extension, and the fact that $h$ is an inclusion means that the composite

$$
\Lambda^{\prime} \stackrel{\varphi}{\rightarrow} \operatorname{Ext}^{1}\left(q, Y^{n} \cap X^{n}\right) \rightarrow \operatorname{Ext}^{1}\left(q, X^{n}\right)
$$

must be injective. Therefore, $\varphi$ must be injective; $\Lambda^{\prime}$ is a subset of $\operatorname{Ext}^{1}\left(q, Y^{n} \cap X^{n}\right)$.

Lemma 3.3. The objects of the category $\mathscr{F}(\mathscr{I}, n)$ all belong to $\mathbf{C}\left(\mathscr{A}^{\mu}\right)=\mathbf{C}(\mathscr{B})$.

Proof. We know that $Y \cap X$ belongs to $\mathscr{I} \subset \mathbf{C}(\mathscr{B})$ and hence all the objects $Y^{i} \cap X^{i}$ belong to $\mathscr{B}$. For $i \neq n, n+1$, we have that $Y^{i}=Y^{i} \cap X^{i} \in \mathscr{B}$. We need to show that $Y^{n}, Y^{n+1} \in \mathscr{B}$. From the pushout square

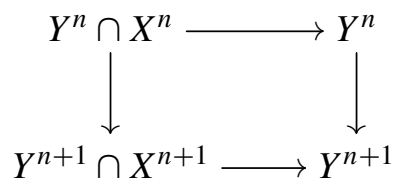

it follows that $Y^{n+1}$ is a quotient of $Y^{n} \oplus\left(Y^{n+1} \cap X^{n+1}\right)$; by Proposition 1.15(ii), $Y^{n+1}$ will belong to $\mathscr{B}$ if $Y^{n}$ does.

In Remark 3.2, we saw that $Y^{n}$ is an extension of $q^{\# \Lambda^{\prime}}$ by $Y^{n} \cap X^{n} \in \mathscr{B}$, where $\Lambda^{\prime}$ can be thought of as a subset $\Lambda^{\prime} \subset \operatorname{Ext}^{1}\left(q, Y^{n} \cap X^{n}\right)$. By Remark 1.17, we know that $\# \Lambda^{\prime} \leq \# \operatorname{Ext}^{1}\left(q, Y^{n} \cap X^{n}\right)<\mu$. But $q$ is the coproduct of the $<\alpha$ quotients $x$ of the generator $g$, all of which belong to $\mathscr{C} \subset \mathscr{B}$; hence, $q^{\# \Lambda^{\prime}}$ is a coproduct of $<\mu$ objects in $\mathscr{B}$ and belongs to $\mathscr{B}$. By Proposition 1.15(iii), $\mathscr{B}$ is closed under extensions, and therefore, $Y^{n}$ also belongs to $\mathscr{B}$. 
Lemma 3.4. The category $\mathscr{g}(\mathscr{I}, n)$ is $\mu$-filtered.

Proof. Since $\mathscr{S}(\mathscr{F}, n)$ is equivalent to a partially ordered set, we need only show that every collection of fewer than $\mu$ objects in $\mathscr{\mathscr { S }}(\mathscr{I}, n)$ is dominated by an object of $\mathscr{f}(\mathscr{g}, n)$. Suppose therefore that we are given a set $\left\{Y_{j} \mid j \in J\right\}$ of $<\mu$ objects of $\mathscr{E}(\mathscr{I}, n)$. The objects $Y_{j} \cap X$ all belong to the $\mu$-filtered category $\mathscr{I}$, and we may therefore choose a $Z \in \mathscr{I}$ dominating them.

Take $k \in \operatorname{Ker}\left(\operatorname{Ext}^{1}\left(q, Z^{n}\right) \rightarrow \operatorname{Ext}^{1}\left(q, X^{n}\right)\right)$. Now $X^{n}=\operatorname{colim}_{X_{i} \in \Phi^{\Phi}} X_{i}^{n}$, the category $\mathscr{I}$ is $\mu$-filtered, and $\operatorname{Ext}^{1}(q,-)$ commutes with $\mu$-filtered colimits. Hence

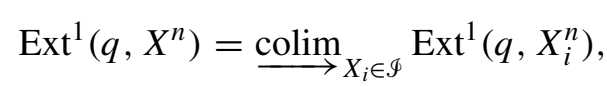

and the fact that $k \in \operatorname{Ext}^{1}\left(q, Z^{n}\right)$ maps to zero in $\underset{\mathscr{c o l i m}}{\longrightarrow} X_{i} \in \mathscr{I} \operatorname{Ext}^{1}\left(q, X_{i}^{n}\right)$ means that we may choose some morphism $Z \rightarrow Z_{k}$ in $\mathscr{I}$ so that $k$ is annihilated by $\operatorname{Ext}^{1}\left(q, Z^{n}\right) \rightarrow \operatorname{Ext}^{1}\left(q, Z_{k}^{n}\right)$. We can choose such a $Z \rightarrow Z_{k}$ for every $k \in$ $\operatorname{Ker}\left(\operatorname{Ext}^{1}\left(q, Z^{n}\right) \rightarrow \operatorname{Ext}^{1}\left(q, X^{n}\right)\right)$. But $\# \operatorname{Ext}^{1}\left(q, Z^{n}\right)<\mu$, and hence, there are $<\mu$ possible $k$. Since $\mathscr{I}$ is $\mu$-filtered, the $Z_{k}$ are all dominated by some object $Z^{\prime} \in \mathscr{I}$. Thus, the map $Z \rightarrow Z^{\prime}$ annihilates all the $k$; on the image $\operatorname{Im}\left(\operatorname{Ext}^{1}\left(q, Z^{n}\right) \rightarrow\right.$ $\left.\operatorname{Ext}^{1}\left(q,\left(Z^{\prime}\right)^{n}\right)\right)$, the map to $\operatorname{Ext}^{1}\left(q, X^{n}\right)$ is injective.

For each $Y_{j}$, we have that $Y_{j}^{n}$ is an extension of $q^{\# \Lambda_{j}}$ by $Y_{j}^{n} \cap X^{n}$, where $\Lambda_{j}$ is a subset of $\operatorname{Ext}^{1}\left(q, Y_{j}^{n} \cap X^{n}\right)$ that maps injectively to $\operatorname{Ext}^{1}\left(q, X^{n}\right)$. We may take the image of $\Lambda_{j}$ under the composite $Y_{j}^{n} \cap X^{j} \rightarrow Z^{n} \rightarrow\left(Z^{\prime}\right)^{n}$ or more precisely under the composite

$$
\operatorname{Ext}^{1}\left(q, Y_{j}^{n} \cap X^{n}\right) \rightarrow \operatorname{Ext}^{1}\left(q, Z^{n}\right) \rightarrow \operatorname{Ext}^{1}\left(q,\left(Z^{\prime}\right)^{n}\right) .
$$

The image of each $\Lambda_{j}$ is contained in $\operatorname{Im}\left(\operatorname{Ext}^{1}\left(q, Z^{n}\right) \rightarrow \operatorname{Ext}^{1}\left(q,\left(Z^{\prime}\right)^{n}\right)\right)$; hence, so is the union of the images $\Lambda^{\prime}=\bigcup \operatorname{Im}\left(\Lambda_{j}\right)$. $\operatorname{But} \operatorname{Im}\left(\operatorname{Ext}^{1}\left(q, Z^{n}\right) \rightarrow \operatorname{Ext}^{1}\left(q,\left(Z^{\prime}\right)^{n}\right)\right)$ maps injectively to $\operatorname{Ext}^{1}\left(q, X^{n}\right)$; and hence, so does its subset $\Lambda^{\prime}$. Let $Y^{\prime}=B\left(Z^{\prime}, \Lambda^{\prime}\right)$.

As in Remark 2.10, for each $y \in \mathscr{A}$, let $T(y)$ be the trivial complex

$$
\cdots \rightarrow 0 \rightarrow 0 \rightarrow y \rightarrow y \rightarrow 0 \rightarrow 0 \rightarrow \cdots
$$

where the nonzero terms are in degrees $n$ and $n+1$. The objects $Y_{j}, Y^{\prime}$, and $B(X, n) \in \mathbf{C}(\mathscr{A})$ fit into extension sequences

$$
\begin{aligned}
& 0 \longrightarrow Y_{j} \cap X \longrightarrow Y_{j} \longrightarrow T(q)^{\# \Lambda_{j}} \longrightarrow 0, \\
& 0 \longrightarrow Z^{\prime} \longrightarrow Y^{\prime} \longrightarrow q^{\# \Lambda^{\prime}} \longrightarrow 0, \\
& 0 \longrightarrow X \longrightarrow X(X, n) \longrightarrow T(q)^{\# \operatorname{Ext}^{1}\left(x, X^{n}\right)} \longrightarrow 0,
\end{aligned}
$$

and the extension classes are all compatible. We may choose maps of extensions 


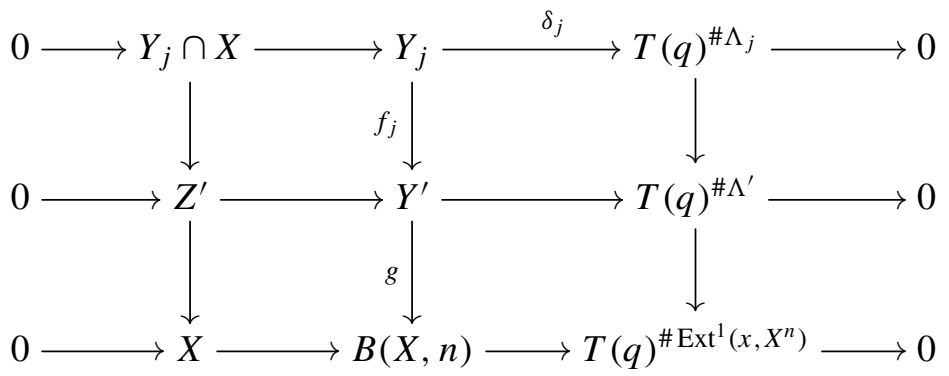

Now the monomorphisms $Y_{j} \cap X \rightarrow Z^{\prime}, Z^{\prime} \rightarrow X, \Lambda_{j} \rightarrow \Lambda^{\prime}$ and $\Lambda^{\prime} \rightarrow \operatorname{Ext}^{1}\left(x, X^{n}\right)$ are all given to us explicitly. The fact that the extension classes are compatible means we may choose maps $f_{j}$ and $g$ as above, but they are not unique. Let us make the choices.

Now each $Y_{j}$ is a subobject of $B(X, n)$; it comes with a given monomorphism $h_{j}: Y_{j} \rightarrow B(X, n)$ making commutative the diagram

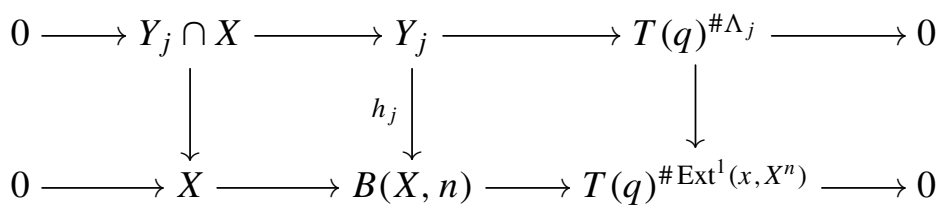

There is no reason to expect that $h_{j}$ should equal $g f_{j}$. The difference $h_{j}-g f_{j}$ must however factor through a map $T(q)^{\# \Lambda_{j}} \rightarrow X$, and maps in $\mathbf{C}(\mathscr{A})$ of the form $T(y) \rightarrow W$ are in bijection with maps $y \rightarrow W^{n}$. Thus, $h_{j}-g f_{j}$ is determined by a map in $\mathscr{A}$ of the form $q^{\# \Lambda_{j}} \rightarrow X^{n}$. But $\operatorname{Hom}\left(q^{\# \Lambda_{j}},-\right)$ commutes with $\mu$ filtered colimits, and $X^{n}$ is the $\mu$-filtered colimit of $X_{i}^{n}, X_{i} \in \mathscr{I}$. For each $j$, we may therefore choose a map $Z^{\prime} \rightarrow Z_{j}$ in $\mathscr{I}$ so that $h_{j}-g f_{j}$ factors through $T(q)^{\# \Lambda_{j}} \rightarrow Z_{j} \subset X$. Since there are fewer than $\mu$ objects $Z_{j} \in \mathscr{I}$, we may find an object $Z^{\prime \prime} \in \mathscr{I}$ dominating them. Let $W=B\left(Z^{\prime \prime}, \Lambda^{\prime}\right)$; that is, form the extension $0 \rightarrow Z^{\prime \prime} \rightarrow W \rightarrow T(q)^{\# \Lambda^{\prime}} \rightarrow 0$ corresponding to the image of $\Lambda^{\prime} \subset \operatorname{Ext}^{1}\left(q,\left(Z^{\prime}\right)^{n}\right)$ under the map $\operatorname{Ext}^{1}\left(q,\left(Z^{\prime}\right)^{n}\right) \rightarrow \operatorname{Ext}^{1}\left(q,\left(Z^{\prime \prime}\right)^{n}\right)$.

Because the extension classes are compatible, we may construct maps of extensions

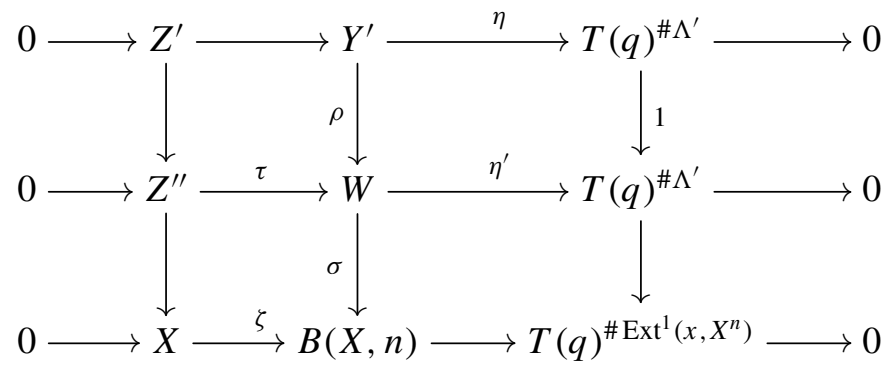


There is no reason to expect $g$ to be equal to $\sigma \rho$, but the difference factors through some $\varphi: T(q)^{\# \Lambda^{\prime}} \rightarrow X$. Changing $\sigma$ to $\sigma+\zeta \varphi \eta^{\prime}$, we achieve that $g=\sigma \rho$. But now we have monomorphisms $Y_{j} \stackrel{f_{j}}{\longrightarrow} Y^{\prime} \stackrel{\rho}{\rightarrow} W \stackrel{\sigma}{\rightarrow} B(X, n)$, and we have that $h_{j}-g f_{j}=h_{j}-\sigma \rho f_{j}$ factors through a map $T(q)^{\# \Lambda_{j}} \stackrel{\theta_{j}}{\longrightarrow} Z^{\prime \prime} \rightarrow X$. Replacing $\rho f_{j}$ by $f_{j}^{\prime}=\rho f_{j}+\tau \theta_{j} \delta_{j}$, we have that $\sigma f_{j}^{\prime}=h_{j}$ for all $j \in J$. Thus, the monomorphisms $h_{j}: Y_{j} \rightarrow B(X, n)$ all factor through $\sigma: W \rightarrow B(X, n)$, and the subobject $\sigma: W \rightarrow$ $B(X, n)$ belongs to $\mathscr{f}(\mathscr{I}, n)$.

Lemma 3.5. $B(X, n)$ is the colimit of its subobjects $Y \in \mathscr{(}(\Phi, n)$.

Proof. Let $Y$ be an object of $\mathscr{G}(\mathscr{I}, n)$. Then we have a monomorphism of short exact sequences

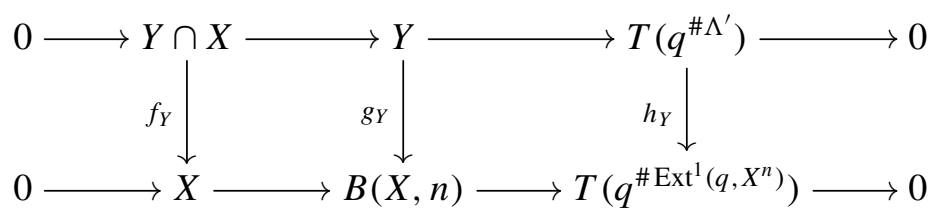

with $h_{Y}$ being the inclusion of a subcoproduct. Since the category $\mathscr{g}(\mathscr{I}, n)$ is filtered, the colimit over $\mathscr{S}(\mathscr{I}, n)$ of the top row is exact; we wish to show that the colimit of $g_{Y}$ is an isomorphism, and the five lemma tells us that it suffices to prove that the colimits of $f_{Y}$ and $h_{Y}$ are isomorphisms. Also, since $f_{Y}$ and $h_{Y}$ are monomorphisms, so are their colimits. It therefore suffices to prove that the colimits of $f_{Y}$ and $h_{Y}$ are epi.

For $f_{Y}$, note that the category $\mathscr{I}$ embeds in the category $\mathscr{I}(\mathscr{I}, n)$; we can view a subobject $Y \subset X$ as a subobject of $B(X, n)$, where the corresponding $\Lambda^{\prime} \subset \operatorname{Ext}^{1}\left(q, Y^{n}\right)$ is empty. But the colimit of $\Phi$ maps epimorphically to $X$, and this epimorphism will factor through the colimit of $f_{Y}$. Hence, the colimit of $f_{Y}$ must be epi.

We need to show that the colimit of $h_{Y}$ is epi. Take any $\lambda \in \operatorname{Ext}^{1}\left(q, X^{n}\right)$; because $\operatorname{Ext}^{1}(q,-)$ commutes with $\mu$-filtered colimits and $X^{n}$ is the $\mu$-filtered colimit of $X_{i}^{n}$, $X_{i} \in \mathscr{I}$, we may choose a $Z \in \mathscr{I}$ and an element $e_{\lambda} \in \operatorname{Ext}^{1}\left(q, Z^{n}\right)$ mapping to $\lambda$. Form the extension $0 \rightarrow Z \rightarrow Y \rightarrow T(q) \rightarrow 0$ corresponding to $e_{\lambda}$. From the fact that $e_{\lambda}$ maps to $\lambda$, we have a map of extensions

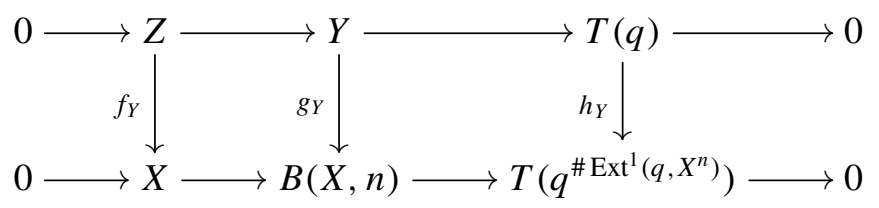

where $h_{Y}$ is the inclusion of the subcoproduct over the singleton $\{\lambda\}$. Thus, the image of the colimit of the $h_{Y}$ must contain the coproduct over every singleton in $\operatorname{Ext}^{1}\left(q, X^{n}\right)$, and hence, it must be epi. 
Lemma 3.6. If filtered colimits of $<\mu$ objects in $\Phi$ belong to $\Phi$, then filtered colimits of $<\mu$ objects in $\mathscr{G}(\mathscr{I}, n)$ belong to $\mathscr{G}(\mathscr{I}, n)$.

Proof. An object $Y$ belongs to $\mathscr{J}(\mathscr{I}, n)$ if it comes with a monomorphism of short exact sequences

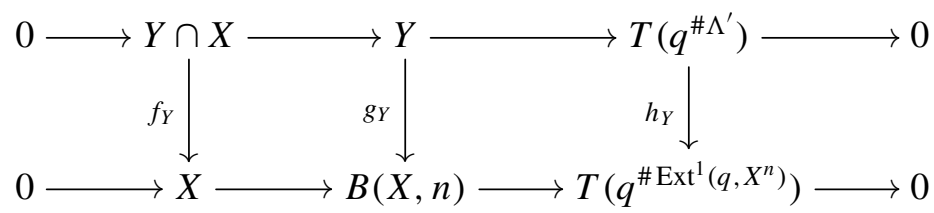

where $h_{Y}$ is the inclusion of a subcoproduct and $Y \cap X \in \mathscr{I}$. A filtered colimit of objects $Y_{\lambda} \cap X, \lambda \in \Lambda$, will belong to $\mathscr{I}$ as long as $\# \Lambda<\mu$ and each $Y_{\lambda} \cap X \in \mathscr{I}$. Filtered colimits are exact, and hence, the filtered colimit of $<\mu$ monomorphisms of short exact sequences as above is such a monomorphism.

Construction 3.7. Recall Remark 2.15: the object $I(X)=J^{\alpha}(X)$ can be constructed using a single sequence. Let us now remember this sequence:

(i) $X_{0}=X$.

(ii) $X_{i+1}=B\left(X_{i}, n\right)$ for some $n$ depending on $i$. The precise relation is that if $i=\ell+m$, where $\ell$ is a limit ordinal and $m$ is an integer, then $n=-m / 2$ if $m$ is even and $n=(m+1) / 2$ if $m$ is odd.

(iii) For limit ordinals $j$ we have $X_{j}={\underset{\text { colim }}{\longrightarrow} i<j}_{i} X_{i}$.

Suppose we are given an $\alpha$-filtered category $\mathscr{I}$ of subobjects of $X$, whose colimit is $X$. For every ordinal $i$, we will now form a subcategory $\Phi_{i}$ of subobjects of $X_{i}$. The rules are:

(i) $\Phi_{0}=\mathscr{I}$.

(ii) If $n$ is the integer for which $X_{i+1}=B\left(X_{i}, n\right)$, then $\mathscr{I}_{i+1}=\mathscr{g}\left(\mathscr{I}_{i}, n\right)$.

(iii) Let $j$ be a limit ordinal. A subobject $Y \subset X_{j}$ belongs to $\mathscr{I}_{j}$ if and only if $Y \cap X_{i}$ belongs to $\Phi_{i}$ for all $i<j$.

Lemma 3.8. Suppose $Y \subset X_{j}$ lies in $\Phi_{j}$ in the notation of Construction 3.7. Then in the triangle $Y \cap X \rightarrow Y \rightarrow A \rightarrow$, we have that $A$ belongs to ${ }^{\perp} \mathbf{K}(\operatorname{Inj} \mathscr{A})$.

Proof. Consider the sequence $Y_{i}=Y \cap X_{i}$. By hypothesis, $Y_{i+1} \in \mathscr{F}\left(\mathscr{I}_{i}, n\right)$, and in Remark 3.2, we saw that $Y_{i+1}=B\left(Y_{i+1} \cap X, \Lambda^{\prime}\right)=B\left(Y_{i}, \Lambda^{\prime}\right)$ for some subset $\Lambda^{\prime} \subset \operatorname{Ext}^{1}\left(q, Y_{i}^{n}\right)$ mapping injectively to $\operatorname{Ext}^{1}\left(q, X^{n}\right)$. And for limit ordinals $\ell$, we have $Y_{\ell}=Y \cap X_{\ell}={\underset{\text { colim }}{\longrightarrow}}_{i<\ell}\left(Y \cap X_{i}\right)=\underline{\text { colim}}_{i<\ell} Y_{i}$. The lemma now follows from Lemma 2.8 and Remark 2.10. 
Lemma 3.9. Let $X$ be an object of $\mathbf{C}(\mathscr{A})$, and let $\mathscr{I} \subset \mathbf{C}(\mathscr{B})=\mathbf{C}\left(\mathscr{A}^{\mu}\right)$ be a full subcategory of the subobjects of $X$. Assume $\mathscr{I}$ is $\mu$-filtered with colimit $X$, and assume that filtered colimits of $<\mu$ objects in $\Phi$ belong to $\Phi$.

Then for every ordinal $i \leq \alpha$, we have that $\Phi_{i}$ has the same properties: it is contained in $\mathbf{C}(\mathscr{B})$, is $\mu$-filtered with colimit $X_{i}$, and is closed under filtered colimits of $<\mu$ objects.

Proof. For $i=0$, we have $\Phi_{0}=\mathscr{I}$ and there is nothing to prove. Suppose the result is true for $i$. By Lemma 3.3, we have $\Phi_{i+1} \subset \mathbf{C}(\mathscr{B})$; by Lemma 3.4, it is $\mu$-filtered; by Lemma 3.5, the colimit is $X_{i+1}$; and by Lemma 3.6, it is closed under filtered colimits of $<\mu$ objects.

For the remainder of the proof, assume $j$ is a limit ordinal and the assertions of the lemma are true for all $i<j$. Let $Y$ be an object of $\mathscr{I}_{j}$. By definition, $Y \cap X_{i} \in \mathscr{I}_{i}$ for every $i<j$, and by induction, $Y \cap X_{i} \in \mathbf{C}(\mathscr{B})$. But $Y={\underset{\operatorname{colim}}{\longrightarrow}}_{i<j}\left(Y \cap X_{i}\right)$ is the colimit of $\leq \alpha<\mu$ objects of $\mathbf{C}(\mathscr{B})$; by Remark 1.16, $Y \in \overrightarrow{\mathbf{C}(\mathscr{B})}$.

Let $Y=\underline{\text { colim}}_{r \in R} Y_{r}$ with $Y_{r} \in \mathscr{I}_{j}$ and $R$ be a filtered category with $<\mu$ objects. For $i<j$, we have that $Y \cap X_{i}={\underset{\text { colim }}{\longrightarrow}}_{r \in R}\left(Y_{r} \cap X_{i}\right)$ belongs to $\mathscr{I}_{i}$ by the induction hypothesis, and hence, $Y \in \mathscr{J}_{j}$ by definition.

Let $\left\{Y_{r} \mid r \in R\right\}$ be a set of $<\mu$ objects of $\Phi_{j}$. By induction on $i<j$, we choose

(i) an object $Z_{0} \in \mathscr{I}_{0}$ containing all the $Y_{r} \cap X_{0}$ and

(ii) an object $Z_{i+1} \in \mathscr{I}_{i+1}$ containing $Z_{i}$ and all the objects $Y_{r} \cap X_{i+1}$.

(iii) For limit ordinals $\ell$, define $Z_{\ell}=\underline{\text { colim }}_{i<\ell} Z_{i}$. Then $Z_{\ell}$ belongs to $\mathscr{I}_{\ell}$ since $Z_{\ell} \cap X_{k}=\underset{\text { colim }}{\longrightarrow} Z_{i<\ell} \cap X_{k}$ belongs to $\mathscr{\Im}_{k}$ for all $k<\ell \leq j$.

But now $Z_{j} \in \mathscr{I}_{j}$ contains all the $Y_{r}$. Thus, $\mathscr{I}_{j}$ is $\mu$-filtered.

The category $\mathscr{I}_{j}$ is a filtered category of subobjects of $X_{j}$, and the colimit is some subobject of $X_{j}$. But it contains the colimits of $\mathscr{I}_{i} \subset \mathscr{I}_{j}$ for all $i<j$; that is, it contains all the $X_{i}$ with $i<j$. Because $X_{j}=$ colim $_{i<j} X_{i}$, we conclude that the colimit of $\mathscr{J}_{j}$ is all of $X_{j}$.

Lemma 3.10. Let $X$ and $\Phi$ be as in Lemma 3.9. The full subcategory of $\Phi_{\alpha}$ whose objects are in $\mathbf{C}(\operatorname{Inj} \mathscr{A})$ is cofinal.

Proof. Let $Y$ be an arbitrary object of $\Phi_{\alpha}$; we need to produce a morphism $Y \rightarrow Z$ in $\mathscr{I}_{\alpha}$ with $Z \in \mathbf{K}(\operatorname{Inj} \mathscr{A})$. We inductively define a sequence $\left\{Z_{i}\right\}$ of objects in $\mathscr{I}_{i}$, and $Z$ will be the colimit; the recipe is:

(i) Put $Z_{0}=Y \cap X$.

(ii) Assume $n$ is the integer for which $\mathscr{I}_{i+1}=\mathscr{I}_{(}\left(\mathscr{I}_{i}, n\right)$, and suppose we have defined $Z_{i} \in \mathscr{I}_{i}$. Choose an object $W_{i} \in \mathscr{I}_{i}$ containing $Z_{i}$ and $Y \cap X_{i}$. There is a morphism $W_{i} \rightarrow V_{i}$ in $\mathscr{I}_{i}$ annihilating the kernel of $\operatorname{Ext}^{1}\left(q, W_{i}^{n}\right) \rightarrow \operatorname{Ext}^{1}\left(q, X_{i}^{n}\right)$; we saw its existence in the proof of Lemma 3.4. Let $\Lambda^{\prime} \subset \operatorname{Ext}^{1}\left(q, V_{i}^{n}\right)$ be the 
image of the map $\operatorname{Ext}^{1}\left(q, W_{i}^{n}\right) \rightarrow \operatorname{Ext}^{1}\left(q, V_{i}^{n}\right)$; then $\Lambda^{\prime}$ maps injectively to $\operatorname{Ext}^{1}\left(q, X_{i}^{n}\right)$, and we can define $Z_{i+1} \in \mathscr{I}_{i+1}$ to be $B\left(V_{i}, \Lambda^{\prime}\right)$. That is, $Z_{i+1}$ is given by a map of extensions

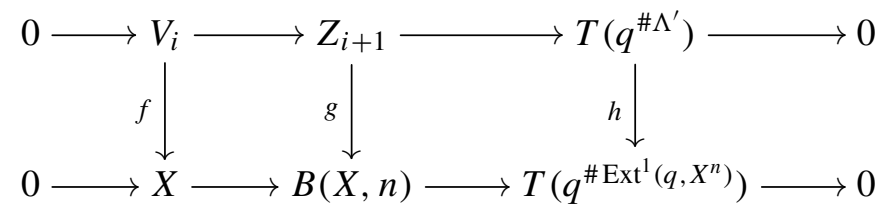

Note that the monomorphisms $f$ and $h$ are given, and we make a choice of a compatible $g$.

(iii) For limit ordinals $\ell$, define $Z_{\ell}={\underset{\text { colim }}{\longrightarrow}}_{i<\ell} Z_{i}$.

We have a map $Z_{i} \rightarrow Z_{i+1}$ that factors as $Z_{i} \rightarrow W_{i} \rightarrow V_{i} \rightarrow Z_{i+1}$. By construction, the map $W_{i} \rightarrow V_{i}$ kills the kernel of the map $\varphi: \operatorname{Ext}^{1}\left(q, W_{i}^{n}\right) \rightarrow \operatorname{Ext}^{1}\left(q, X_{i}^{n}\right)$ while the morphism $V_{i} \rightarrow Z_{i+1}$ kills the image of $\varphi$. It follows that the composite $Z_{i}^{n} \rightarrow$ $Z_{i+1}^{n}$ is annihilated by $\operatorname{Ext}^{1}(q,-)$ for the choice of $n$ for which $X_{i+1}=B\left(X_{i}, n\right)$.

The $n$ are chosen so that, for any limit ordinal $i$, every integer $n$ occurs between $i$ and $i+\omega$. If we restrict to limit ordinals, we have that $\operatorname{Ext}^{1}(q,-)$ annihilates $Z_{i}^{n} \rightarrow Z_{j}^{n}$ for any integer $n$ and any pair $i<j$ of limit ordinals. But $Z_{\alpha}$ is the $\alpha$-filtered colimit of the limit ordinals $<\alpha$, and for each quotient $x$ of the generator $g$, we have that $\operatorname{Ext}^{1}(x,-)$ commutes with $\alpha$-filtered colimits. It follows that $\operatorname{Ext}^{1}\left(x, Z_{\alpha}^{n}\right)=0$ for all $x$ and all $n$, and hence, $Z_{\alpha}$ is a complex of injectives. And by construction, $Y=\underset{\operatorname{colim}}{\longrightarrow}\left(Y \cap X_{i}\right)$ maps in $\Phi_{\alpha}$ to $Z=\underset{\text { colim }}{\longrightarrow} Z_{i}$.

Corollary 3.11. Let $Y \in \mathscr{I}_{\alpha}$ be in the cofinal subcategory of objects that lie in $\mathbf{K}(\operatorname{Inj} \mathscr{A})$. Then $Y=I(Y \cap X)$.

Proof. By Lemma 3.8, the triangle $Y \cap X \rightarrow Y \rightarrow A \rightarrow$ has $A \in{ }^{\perp} \mathbf{K}(\operatorname{Inj} \mathscr{A})$. Since $Y$ belongs to $\mathbf{K}(\operatorname{Inj} \mathscr{A})$, the triangle identifies $Y$ with $I(Y \cap X)$.

Lemma 3.12. Let I $: \mathbf{C}(\mathscr{A}) \rightarrow \mathbf{C}(\operatorname{Inj} \mathscr{A})$ be the functor of Theorem 2.13. Then the objects $\{I(s) \mid s \in \mathbf{C}(\mathscr{B})\}$ generate the triangulated category $\mathbf{K}(\operatorname{Inj} \mathscr{A})$.

Proof. For every nonzero object $X \in \mathbf{K}(\operatorname{Inj} \mathscr{A})$, we need to produce a nonzero map $I(s) \rightarrow X$ in $\mathbf{K}(\operatorname{Inj} \mathscr{A})$ or equivalently (by the fact that $I$ is left-adjoint to the inclusion) a nonzero map $s \rightarrow X$ in $\mathbf{K}(\mathscr{A})$. The proof is as in [Krause 2001, Lemma 2.2].

If $X$ is not acyclic, there is a nontrivial cohomology group; without loss, we may assume $H^{0}(X) \neq 0$. Let $K \subset X^{0}$ be the kernel of $\partial: X^{0} \rightarrow X^{1}$; we may choose a map $g \rightarrow K$ that does not factor through the image of $X^{-1} \rightarrow X^{0}$. But then $g \rightarrow K \rightarrow X^{0}$ extends to a chain map $g \rightarrow X$ that is nonzero in homology, and $g$ is $\mu$-presentable; that is, $g \in \mathbf{C}(\mathscr{B})$. 
It remains to handle the case where $X$ is acyclic. If $X$ is nonzero in $\mathbf{K}(\operatorname{Inj} \mathscr{A})$, then it is not a contractible complex, so there must be an $n$ for which $\operatorname{Im}\left(X^{n} \rightarrow X^{n+1}\right)$ is not an injective object of $\mathscr{A}$. Suppose without loss that $M=\operatorname{Im}\left(X^{-2} \rightarrow X^{-1}\right)$ is not injective. Then there is a quotient $x$ of the generator $g$ and a nonzero element of $\operatorname{Ext}^{1}(x, M)$. But elements of $\operatorname{Ext}^{1}(x, M)$ are in bijection with morphisms $x \rightarrow X$ in $\mathbf{K}(\mathscr{A})$, so we have produced a nonzero map $x \rightarrow X$ where $x$ is $\mu$-presentable, that is, $x \in \mathbf{C}(\mathscr{B})$.

Theorem 3.13. Let $\mathscr{B} \subset \mathscr{A}$ be as in Definition 1.13; by Proposition 1.18, it is precisely the category $\mathscr{B}=\mathscr{A}^{\mu}$ of $\mu$-presentable objects in $\mathscr{A}$. Then the objects $\{I(s) \mid s \in \mathbf{C}(\mathscr{B})\}$ form a $\mu$-compact generating set in the category $\mathbf{K}(\operatorname{Inj} \mathscr{A})$. Therefore, $\mathbf{K}(\operatorname{Inj} \mathscr{A})$ is well generated.

Proof. The fact that these objects generate was proved in Lemma 3.12; what remains is to show that they form a $\mu$-compact generating set, meaning that they form a $\mu$-perfect set of $\mu$-small objects; see [Neeman 2001, §3.3, §4.1, and §4.2]. Suppose we are given a set $\left\{X_{\lambda} \mid \lambda \in \Lambda\right\}$ of objects of $\mathbf{K}(\operatorname{Inj} \mathscr{A})$. Then the coproduct of these objects in $\mathbf{K}(\operatorname{Inj} \mathscr{A})$ is formed by applying the functor $I$ of Theorem 2.13 to the ordinary coproduct in $\mathbf{K}(\mathscr{A})$ or $\mathbf{C}(\mathscr{A})$. But now, in the category $\mathbf{C}(\mathscr{A})$, each $X_{\lambda}$ is the $\mu$-filtered colimit of its subobjects $\left\{s \rightarrow X_{\lambda} \mid s \in \mathbf{C}(\mathscr{B})\right\}$, and the coproduct of the $X_{\lambda}$ satisfies

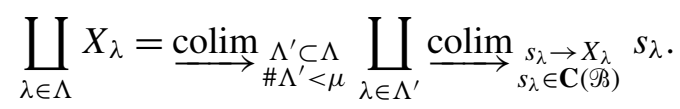

Thus, we wish to apply our lemmas to the object $X=\bigsqcup X_{\lambda}$ and to the category $\mathscr{I}$ consisting of subobjects $\bigsqcup_{\lambda \in \Lambda^{\prime}} s_{\lambda}$, where $\Lambda^{\prime} \subset \Lambda$ is a set with $\# \Lambda^{\prime}<\mu$ and each $s_{\lambda} \in \mathbf{C}(\mathscr{B})$ is a subobject of $X_{\lambda}$.

We have proved that $I(X)=X_{\alpha}$ is the colimit in $\mathbf{C}(\mathscr{A})$ of the $\mu$-filtered category $\Im_{\alpha}$, and hence, any map from the $\mu$-presentable $s \in \mathbf{C}(\mathscr{B})$ to $I(X)$ must factor through some object $Y \in \Phi_{\alpha}$. By Lemma 3.8, $Y$ fits in a triangle $Y \cap X \rightarrow Y \rightarrow A \rightarrow$ with $A \in{ }^{\perp} \mathbf{K}(\operatorname{Inj} \mathscr{A})$; if we apply the functor $I$, then it takes $Y \cap X \rightarrow Y$ to an isomorphism. Thus, the map $s \rightarrow I(X)$ factors as $s \rightarrow I(Y \cap X) \rightarrow I(X)$, and $Y \cap X$ is an object of $\mathscr{I}$, meaning a coproduct $\bigsqcup_{\lambda \in \Lambda^{\prime}} s_{\lambda}$, where $\Lambda^{\prime} \subset \Lambda$ is a set with $\# \Lambda^{\prime}<\mu$ and each $s_{\lambda} \in \mathbf{C}(\mathscr{B})$ is a subobject of $X_{\lambda}$. In the category $\mathbf{K}(\operatorname{Inj} \mathscr{A})$, we have factored the map as

$$
I(s) \rightarrow I\left(\coprod_{\lambda \in \Lambda^{\prime}} s_{\lambda}\right) \rightarrow I\left(\coprod_{\lambda \in \Lambda} X_{\lambda}\right) .
$$

Now suppose that we are given in $\mathbf{K}(\operatorname{Inj} \mathscr{A})$ a vanishing composite

$$
I(s) \stackrel{\theta}{\rightarrow} I\left(\coprod_{\lambda \in \Lambda^{\prime}} s_{\lambda}\right) \stackrel{\sigma}{\rightarrow} I\left(\coprod_{\lambda \in \Lambda} X_{\lambda}\right),
$$


that is, we are given a map $\theta$ so that $\sigma \theta$ is null-homotopic. Let us write this a little more compactly: we are given a morphism $\theta: I(s) \rightarrow I(W)$ so that the composite $I(s) \stackrel{\theta}{\rightarrow} I(W) \stackrel{\sigma}{\rightarrow} I(X)$ is null-homotopic with $W=\bigsqcup_{\lambda \in \Lambda^{\prime}} s_{\lambda}$ belonging to $\mathscr{I}=\mathscr{I}_{0}$. Of course, we are free to replace $W$ by a larger subobject in $\mathscr{I}$ before proceeding any further, and Lemma 3.10 tells us that in the category $\Phi_{\alpha}$ the objects that belong to $\mathbf{C}(\operatorname{Inj} \mathscr{A})$ are cofinal. We may therefore produce in $\Phi_{\alpha}$ a map $W \rightarrow Y$ with $Y$ in the subcategory. We have a morphism $s \rightarrow I(s) \rightarrow I(Y)=Y$ in $\mathbf{K}(\operatorname{Inj} \mathscr{A})$; choose a representative, that is, a chain map $s \rightarrow Y$ in $\mathbf{C}(\mathscr{A})$. We know that the composite $s \rightarrow Y \rightarrow I(X)$ is null-homotopic.

But $I(X)$ is the $\mu$-filtered colimit of $\Phi_{\alpha}$, and $s$ is $\mu$-presentable. There is a map $Y \rightarrow Z$ in $\Phi_{\alpha}$ so that the composite $s \rightarrow Y \rightarrow Z$ is already null-homotopic. Now recalling that the maps $I(Y \cap X) \rightarrow I(Y)=Y$ and $I(Z \cap X) \rightarrow I(Z)$ are isomorphisms in $\mathbf{K}(\operatorname{Inj} \mathscr{A})$, we have proved that for some $Z \in I_{\alpha}$ the map $s \rightarrow I(Y \cap X) \rightarrow I(Z \cap X)$ is zero in $\mathbf{K}(\operatorname{Inj} \mathscr{A})$, that is, we have proved the vanishing of some

$$
I(s) \stackrel{\theta}{\rightarrow} I\left(\coprod_{\lambda \in \Lambda^{\prime}} s_{\lambda}\right) \rightarrow I\left(\coprod_{\lambda \in \Lambda^{\prime \prime}} t_{\lambda}\right)
$$

\section{The failure of recollement}

In the generality where $\mathscr{A}$ is any Grothendieck abelian category, we have natural functors

$$
\mathbf{K}_{\mathrm{ac}}(\operatorname{Inj} \mathscr{A}) \stackrel{J}{\rightarrow} \mathbf{K}(\operatorname{Inj} \mathscr{A}) \stackrel{Q}{\rightarrow} \mathbf{D}(\mathscr{A})
$$

that compose to zero. But the functor $Q$ has a right adjoint $Q_{\rho}$, namely the functor taking $X \in \mathbf{D}(\mathscr{A})$ to its $K$-injective injective resolution. Since $Q_{\rho}$ is fully faithful, the map $Q$ must be a Verdier quotient, but $J$ is precisely the inclusion of the kernel of $Q$. It therefore follows that $J$ also has a right adjoint $J_{\rho}$.

Krause [2005] proves that, provided the category $\mathscr{A}$ is locally noetherian and $D(\mathscr{A})$ is compactly generated, then $J$ and $Q$ have left adjoints as well. In particular, $J$ takes products to products: products of acyclic complexes of injectives are acyclic. What we will now produce is:

Example 4.1. There is a locally noetherian abelian category $\mathscr{A}$ for which $\mathbf{K}(\operatorname{Inj} \mathscr{A})$ is not closed under products. The category $\mathscr{A}$ will be (a special case of) the category $\mathscr{A}$ of [Neeman 2011, Construction 1.1]; the counterexample works in the generality of the $\mathscr{A}$ of [Neeman 2011, Construction 1.1], but for simplicity, we will specialize to a particular case. And the chain complex of injectives will be a minor modification of the chain complex of [Neeman 2011, proof of Theorem 1.1, pp. 830-831].

Let $k$ be a field, and let $R_{1}=R$ be the ring $k[x] /\left(x^{2}\right)$ of dual numbers over $k$. The ring $R_{n}$ is $R \otimes_{k} R \otimes_{k} \cdots \otimes_{k} R$, the tensor product of $n$ copies of $R$. The inclusions 
$R_{n} \rightarrow R_{n+1}$ are the inclusions into the first $n$ factors. And $S$ is the colimit of $R_{n}$. If we write $S$ as

$$
S=\frac{k\left[x_{1}, x_{2}, x_{3}, \ldots\right]}{\left(x_{1}^{2}, x_{2}^{2}, x_{3}^{2}, \ldots\right)},
$$

then $\mathscr{A}$ is the category of all $S$-modules $M$, where each element $m \in M$ is annihilated by all but finitely many of the $x_{i}$.

Let $B$ be an injective resolution of $\Sigma k$ over the ring $R$; for definiteness, let us choose $B$ to be the complex starting in degree -1

$$
\cdots \rightarrow 0 \rightarrow 0 \rightarrow R \stackrel{x}{\rightarrow} R \stackrel{x}{\rightarrow} \cdots .
$$

Let $C_{n}$ be the complex $B^{\otimes n}$, that is, the tensor product of $n$ copies of $B$. Then $C_{n}$ is an injective resolution of $\Sigma^{n} k$ over the ring $R_{n}=k\left[x_{1}, x_{2}, \ldots, x_{n}\right] /\left(x_{1}^{2}, x_{2}^{2}, \ldots, x_{n}^{2}\right)$. Consider the chain map $k \rightarrow B$, which takes $1 \in k$ to $x \in R=B^{0}$, that is, the chain map

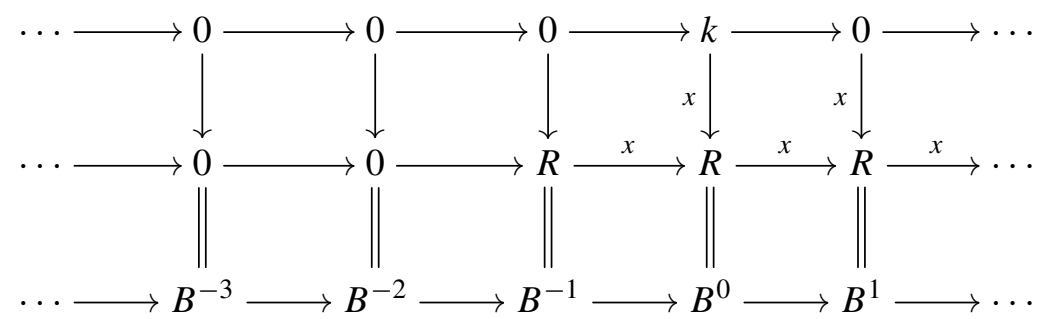

We have an induced inclusion $C_{n}=C_{n} \otimes_{k} k \rightarrow C_{n} \otimes_{k} B=C_{n+1}$, and we define $C$ to be the colimit of the $C_{n}$. Then $C$ is an acyclic complex of injective objects in $\mathscr{A}$.

Now let $c_{n} \in C^{0}$ be the cycle $x \otimes x \otimes x \otimes \cdots$, which we view as $x_{1} \otimes x_{2} \otimes x_{3} \otimes \cdots$; the only question is which degree each $x_{i}=x \in R$ lives in. The rule is: for $1 \leq i \leq n$, we have $x_{i} \in B^{-1}=R$; for $n+1 \leq i \leq 2 n$, we have $x_{i} \in B^{1}=R$; and for $2 n<i$, we put $x_{i} \in B^{0}=R$. Note that $x_{1} \otimes x_{2} \otimes \cdots \otimes x_{n} \in C_{n}^{-n}$ is not a boundary; it defines the unique nonvanishing cohomology class of $C_{n} \cong \Sigma^{n} k$. Of course, $c_{n} \in C$ is a cycle in the acyclic complex $C$ and hence a boundary, but it must be a boundary of some chain in $C^{-1}$ that nontrivially involves the terms in the tensor product with $i>n$; in other words, if $c_{n}$ is the boundary of a chain $b_{n} \in C$, then there exists an $i>n$ so that $x_{i} b_{n} \neq 0$. The product $\prod_{n=1}^{\infty} c_{n}$ is a product of cycles in $C^{0}$ and hence is a cycle in the complex $\prod_{n=0}^{\infty} C$. But it cannot be a boundary; if it were the boundary of $\prod_{n=1}^{\infty} b_{n}$, we would have infinitely many $i$ and infinitely many $n_{i}$ for which $x_{i} b_{n_{i}} \neq 0$, meaning $\prod_{n=1}^{\infty} b_{n}$ cannot belong to the category $\mathscr{A}$.

Remark 4.2. Since the category $\mathscr{A}$ is locally noetherian, it follows that its derived category $\mathbf{D}(\mathscr{A})$ cannot possibly be compactly generated. After all, Krause [2005] proved that when $D(\mathscr{A})$ is compactly generated then $\mathbf{K}(\operatorname{Inj} \mathscr{A})$ is closed under products. 


\section{References}

[Bican et al. 2001] L. Bican, R. El Bashir, and E. Enochs, "All modules have flat covers", Bull. London Math. Soc. 33:4 (2001), 385-390. MR 2002e:16002 Zbl 1029.16002

[Gabriel and Ulmer 1971] P. Gabriel and F. Ulmer, Lokal präsentierbare Kategorien, Lecture Notes in Mathematics 221, Springer, Berlin, 1971. MR 48 \#6205 Zbl 0225.18004

[Iyengar and Krause 2006] S. Iyengar and H. Krause, "Acyclicity versus total acyclicity for complexes over Noetherian rings”, Doc. Math. 11 (2006), 207-240. MR 2007h:16013 Zbl 1119.13014

[Jørgensen 2005] P. Jørgensen, “The homotopy category of complexes of projective modules”, Adv. Math. 193:1 (2005), 223-232. MR 2005m:18013 Zbl 1068.18012

[Krause 2001] H. Krause, “On Neeman's well generated triangulated categories”, Doc. Math. 6 (2001), 121-126. MR 2002c:18008 Zbl 0987.18012

[Krause 2005] H. Krause, "The stable derived category of a Noetherian scheme", Compos. Math. 141:5 (2005), 1128-1162. MR 2006e:18019 Zbl 1090.18006

[Krause 2012] H. Krause, "Approximations and adjoints in homotopy categories", Math. Ann. 353:3 (2012), 765-781. MR 2923949 Zbl 06050576

[Murfet 2007] D. S. Murfet, The mock homotopy category of projectives and Grothendieck duality, Ph.D. thesis, Australian National University, 2007, Available at http://therisingsea.org/notes/ thesis.pdf.

[Neeman 2001] A. Neeman, Triangulated categories, Annals of Mathematics Studies 148, Princeton University Press, 2001. MR 2001k:18010 Zbl 0974.18008

[Neeman 2008] A. Neeman, "The homotopy category of flat modules, and Grothendieck duality", Invent. Math. 174:2 (2008), 255-308. MR 2009h:16008 Zbl 1184.18008

[Neeman 2010] A. Neeman, "Some adjoints in homotopy categories", Ann. of Math. (2) 171:3 (2010), 2143-2155. MR 2011i:18026 Zbl 1205.18008

[Neeman 2011] A. Neeman, "Non-left-complete derived categories", Math. Res. Lett. 18:5 (2011), 827-832. MR 2875857 Zbl 1244.18009

Communicated by David Benson

Received 2013-03-14 Revised 2013-08-06 Accepted 2013-09-09

amnon.neeman@anu.edu.au

Centre for Mathematics and its Applications, Mathematical Sciences Institute, Australian National University, John Dedman Building 27, Canberra ACT 0200, Australia 


\section{Algebra \& Number Theory}

msp.org/ant

\section{EDITORS}

MANAGING EDITOR

Bjorn Poonen

Massachusetts Institute of Technology

Cambridge, USA

\author{
EDITORIAL BOARD CHAIR \\ David Eisenbud \\ University of California \\ Berkeley, USA
}

\section{BOARD OF EDITORS}

Georgia Benkart

Dave Benson

Richard E. Borcherds

John H. Coates

J-L. Colliot-Thélène

Brian D. Conrad

Hélène Esnault

Hubert Flenner

Edward Frenkel

Andrew Granville

Joseph Gubeladze

Roger Heath-Brown

Ehud Hrushovski

Craig Huneke

Mikhail Kapranov

Yujiro Kawamata

János Kollár

Yuri Manin

Barry Mazur

Philippe Michel
University of Wisconsin, Madison, USA

University of Aberdeen, Scotland

University of California, Berkeley, USA

University of Cambridge, UK

CNRS, Université Paris-Sud, France

University of Michigan, USA

Freie Universität Berlin, Germany

Ruhr-Universität, Germany

University of California, Berkeley, USA

Université de Montréal, Canada

San Francisco State University, USA

Oxford University, UK

Hebrew University, Israel

University of Virginia, USA

Yale University, USA

University of Tokyo, Japan

Princeton University, USA

Northwestern University, USA

Harvard University, USA

École Polytechnique Fédérale de Lausanne
Susan Montgomery

Shigefumi Mori

Raman Parimala

Jonathan Pila

Victor Reiner

Karl Rubin

Peter Sarnak

Joseph H. Silverman

Michael Singer

Vasudevan Srinivas

J. Toby Stafford

Bernd Sturmfels

Richard Taylor

Ravi Vakil

Michel van den Bergh

Marie-France Vignéras

Kei-Ichi Watanabe

Efim Zelmanov

Shou-Wu Zhang
University of Southern California, USA

RIMS, Kyoto University, Japan

Emory University, USA

University of Oxford, UK

University of Minnesota, USA

University of California, Irvine, USA

Princeton University, USA

Brown University, USA

North Carolina State University, USA

Tata Inst. of Fund. Research, India

University of Michigan, USA

University of California, Berkeley, USA

Harvard University, USA

Stanford University, USA

Hasselt University, Belgium

Université Paris VII, France

Nihon University, Japan

University of California, San Diego, USA

Princeton University, USA

PRODUCTION

production@msp.org

Silvio Levy, Scientific Editor

See inside back cover or msp.org/ant for submission instructions.

The subscription price for 2014 is US $\$ 225 /$ year for the electronic version, and $\$ 400 /$ year $(+\$ 55$, if shipping outside the US) for print and electronic. Subscriptions, requests for back issues and changes of subscribers address should be sent to MSP.

Algebra \& Number Theory (ISSN 1944-7833 electronic, 1937-0652 printed) at Mathematical Sciences Publishers, 798 Evans Hall \#3840, c/o University of California, Berkeley, CA 94720-3840 is published continuously online. Periodical rate postage paid at Berkeley, CA 94704, and additional mailing offices.

ANT peer review and production are managed by EditFLOW ${ }^{\circledR}$ from Mathematical Sciences Publishers.

\section{PUBLISHED BY}

- mathematical sciences publishers

nonprofit scientific publishing

http://msp.org/

(C) 2014 Mathematical Sciences Publishers 


\section{Algebra \& Number Theory}

Volume $8 \quad$ No. $2 \quad 2014$

Large self-injective rings and the generating hypothesis

LEIGH SHEPPERSON and NEIL STRICKLAND

On lower ramification subgroups and canonical subgroups

303

SHIN HATTORI

Wild models of curves

DINO LORENZINI

Geometry of Wachspress surfaces

COREY IRVING and HAL SCHENCK

Daniel Goldstein, Robert M. GuRAlnick, Mark L. Lewis, AleXander Moretó, Gabriel Navarro and Pham HuU TieP

The homotopy category of injectives

AMNON NEEMAN

Essential dimension of spinor and Clifford groups

Vladimir Chernousov and AleXANDER MERKurJeV

On Deligne's category $\underline{\operatorname{Rep}}^{a b}\left(S_{d}\right)$

JONATHAN COMES and VICTOR OSTRIK

Algebraicity of the zeta function associated to a matrix over a free group algebra

CHRISTIAN KASSEL and Christophe REUTENAUER 